\title{
Reactive Nitrogen Species as Therapeutic Targets for Autophagy: Implication for Ischemic Stroke
}

Authors' information:

1. Jinghan Feng a,b M. Chin. Med. Tel.:852-6560-2480 Fax:852-2168-4259 Email: jinghanf@hku.hk

2. Xingmiao Chen ${ }^{\mathrm{a}, \mathrm{b}}$ PhD Tel.:852-6708-9985 Fax: 852-2168-4259

Email: chenxm@hku.hk

3. Jiangang Shen ${ }^{\text {a,b }}$ M.D., Prof. Tel.:852-3917-6429 Fax: 852-2168-4259 Email: shenjg@hku.hk

a. School of Chinese Medicine, LKS Faculty of Medicine, The University of Hong Kong, Hong Kong SAR, China

b. The University of Hong Kong-Shenzhen Institute of Research and Innovation (HKU-SIRI), Shenzhen, China

Corresponding should be addressed to:

Dr. Jiangang Shen, School of Chinese Medicine, LKS Faculty of Medicine, The University of Hong Kong, No.10 Sassoon Road, Pokfulam, Hong Kong, China.

Tel.: +852-3917-6429

Fax: $+852-2168-4259$

E-mail: shenjg@hku.hk. 


\section{Abstract}

Introduction: Roles of autophagy/mitophagy activation in ischemic stroke remain controversial. To elucidate potential reasons, we analyze the factors responsible for divergent results in literatures. Reactive nitrogen species (RNS) are important cytotoxic factors in ischemic stroke. Herein, we particularly discuss the roles played by RNS in autophagy/mitophagy and ischemic brain injury.

Areas covered: Following factors should be considered in the studies on autophagy/mitophagy in ischemic stroke: (1) Protocols for administration of autophagy regulators including administration time points, routes and doses, etc.; (2) Specificity of autophagy regulators; (3) Animal models of cerebral ischemia with or without reperfusion. In the underlying mechanisms of autophagy/mitophagy, we particularly discuss the potential roles of RNS in mediating excessive autophagy/mitophagy during cerebral ischemia/reperfusion injury.

Expert opinion: Emphasis should be given to the following aspects in future studies:

(1) Targeting RNS and related cellular signaling pathways in the regulation of autophagy/mitophagy might be a promising strategy for developing novel drugs as well as combined therapy for thrombolytic treatment to reach better outcomes for ischemic stroke; (2) Developing circulating plasma biomarkers linking RNS-mediated autophagy/mitophagy to the magnitude of ischemic brain injury will benefit for stroke treatment. Subsequently, RNS could be dominant therapeutic targets to regulate autophagy/mitophagy for ischemic stroke. 
Key words: Autophagy; ischemic stroke; mitochondria; mitophagy; programmed cell death; reactive nitrogen species.

\section{Introduction}

Autophagy is a sophisticated catabolic process in which cytosolic contents and organelles are transported to lysosomes for degradation.[1] Autophagic process is commonly sub-typed into microautophagy, chaperone-mediated autophagy and marcoautophagy. In this article, we mainly discuss macroautophagy (hereafter referred to as autophagy), a major autophagic type for cellular self-regulation. Autophagy was firstly defined by C de Duve in 1960s, nearly ten years after his discovery of "lysosome" in rat livers.[2] Distinct from microautophagy and chaperone-mediated autophagy, autophagy is characterized as an evolutionary conserved process of 'self-eating' to digest defective or aggregated proteins and organelles via the formation of an autophagosome and its fusion with a lysosome.[3] Furthermore, autophagy also serves as a recycling process to support cell survival by reserving biosynthetic materials derived from degradation as 'building block' under energy shortage and nutrient starvation.[4] Autophagic process is a sophisticated intracellular regulating mechanism depending on a complex network of different autophagy-related (Atg) proteins. These Atg proteins participate in different phases of autophagy.[2] Clearly, autophagy is an ancient but sophisticated self-regulation to keep physiological function and defense unexpected stress and stimulus.

Autophagy is a critical player in various physiological progress including cellular 
growth, differentiation and homeostasis through continually turnover of dysfunctional proteins and organelles. Autophagic process involves initiation/induction, autophagosome formation and maturation/recycle.[5] Briefly, a complete autophagic process can be summarized into several steps: (1) Sequestration: formation of phagophors and sequestrating cytoplasmic constituents into autophagosomes; (2) Maturation: fusion of autophagosomes with lysosomes; (3) Degradation: degradation of autophagosomes by lysosomal hydrolases; (4) Recycle: reusing degraded cytoplasmic materials. With the 'self-eating' process, the damaged cellular components are degraded for cell survival under various stress conditions like oxidative stress, endoplasmic reticulum (ER) stress and starvation, etc.

Central nerve system (CNS) is comparatively more vulnerable to microenvironment disruption than other organs. Emphasis is given to autophagy as a wise sweeper in the brain to maintain CNS's physiological function.[6] Thus, CNS requires more rigorous quality control system including blood brain barrier (BBB) and autophagy for maintaining chemical hemostasis and quickly removing waste products as well as adapting its high demand of energy.[5] Emerging evidences point out the important roles of autophagy in neurodegenerative diseases (NDDs), including Alzheimer's disease [7], Parkinson's disease [8], Huntington's disease [9], and amyotrophic lateral sclerosis [10]. Dysregulation of autophagy in NDDs has been intensively studied and reviewed in literatures $[11,12]$ and targeting autophagy becomes a promising strategy for seeking therapeutic drug candidates for NDDs [13]. Obviously, autophagy becomes a hot research field in NDDs. 
Autophagy is also an important pathophysiological process in stroke. The roles of autophagy-lysosomal system in hemorrhagic stroke have been discussed in a recent review article.[14] Herein, we mainly discuss the roles and underlying mechanisms of autophagy in ischemic stroke. Autophagic flux can be triggered by ischemia stress, but its roles in ischemic stroke is still controversial.[15] Autophagy can be classified into basal and induced autophagy: Basal autophagy is a 'housekeeping' process in neurons [16] whereas induced autophagy possesses double-edged effects on neurons [17]. Multiple autophagic protein markers such as Beclin 1 and LC3-phosphatidylethanolamine conjugate (LC3-II) were found to be robustly increased in cerebral ischemia animal models.[18] Also, autophagososmes and autolysosomes were directly found in ischemic areas by transmission electron microscopy.[19, 20] However, the roles of autophagy in ischemic brain injury remain controversial. Series of investigations indicate that autophagy like a double-edged sword, possesses both beneficial and detrimental effects to the brain after ischemia stimulus.[21]

Recent progress draws an attention to the roles of free radicals in regulating autophagy in ischemic stroke. Redox homeostasis is essential for maintaining physiological functions through an equilibrium between antioxidant and oxidant levels. Imbalance of redox often results in oxidative/nitrosative stress which mediates dysregulation of autophagy, leading to pathological process in neurodegeneration.[22] Free radicals including reactive oxygen species (ROS) and reactive nitrogen species (RNS) are major pro-oxidants during oxidative/nitrosative stress. Among them, 
superoxide anions $\left(\mathrm{O}_{2}{ }^{--}\right)$is a precursor for other types of ROS, including hydrogen peroxide, hydroxyl radical, hypochlorous acid, and hydroperoxyl radical. Besides, nitric oxide (NO) and peroxynitrite anion $\left(\mathrm{ONOO}^{-}\right)$are two representative species of RNS. NO is mainly produced from L-arginine and oxygen catalyzed by various nitric oxide synthases (NOS). In presence of $\mathrm{O}_{2}{ }^{--}$, $\mathrm{NO}$ can rapidly react with $\mathrm{O}_{2}{ }^{--}$to generate $\mathrm{ONOO}^{-} \cdot[23,24]$ Ischemia insults initiate to produce a large amount of free radicals and result in neuronal cell death, BBB disruption and neurological deficits.[25] Growing evidences suggest that ROS can mediate autophagy via posttranslational modification of biomolecules including proteins, lipids and DNA in ischemic stroke.[26, 27] However, the roles and underlying mechanisms of RNS-mediated autophagy in ischemic stroke have yet received enough attention.

In this article, we review current progress regarding the roles of autophagy in the pathological process of cerebral ischemia/reperfusion (I/R) injury. In particular, we emphasize on the roles of RNS in regulating autophagy and their potential impacts on developing novel therapeutic approaches for ischemic stroke.

\section{Autophagy in ischemic stroke}

\subsection{Detection of autophagy activation in ischemic brain}

Autophagy can be evaluated through the detection of multiple autophagic markers in each step or direct morphology observation of autolysosomes.[20] Increased expression of Beclin 1 and LC3-II often serve as useful markers to indicate autophagosomes formation. Especially, labeling LC3 is commonly applied to track 
autophagosomes by observing its distribution change from uniform residence to punctate dots.[28] Moreover, p62/SQSTM1 has multiple domains mediating its interaction with relevant binding partners and functions as an autophagy receptor for selective degradation of ubiqutinated substrates.[29] Accompanying with the degradation of substrates, p62/SQSTM1 is also digested within autolysosomes. Thus, decreased p62/SQSTM1 level could be an indicator for autophagy maturation and degradation.[30] LC3 and p62/SQSTM1 have been documented to be specific and reliable autophagy markers in formalin fixed and paraffin embedded human tissues for immunohistochemistry.[31] With the progress of the mechanisms, we believe that more and more specific autophagy biomarkers would be discovered in future.

The activation of autophagy in ischemic stroke has been intensively studied. Autophagic flux was not only reported in ischemic brains of experimental stroke rodent models with morphological evidences and changes of autophagic markers [32], but also in the brains of ischemic stroke patients with increased levels of Beclin 1 and LC3 in cerebrospinal fluid and serum [33]. The appearance of autophagic vesicles has been also found in human post-mortem brain tissue after stroke.[34] After ischemic insults, autophagic flux not only occurs in neurons [35], but also astrocytes [36] and endothelial cells [37]. Thus, autophagy could be a critical player in the pathological process of ischemic brain injury which involves multiple cells at different stages of ischemic stroke.

\subsection{Controversial roles of activated autophagy in ischemic brain injury}

No consensus about the roles of activated autophagy in ischemic stroke is documented 
in current stage of the investigations. Yet, two things are irrefutable. One is that increased autophagy occurs in the brain in response to ischemic injury; the other is that basal level autophagy is indispensable for keeping brain function. Since these opinions are widely discussed elsewhere, we will not further elaborate here.[21, 38] It has been an intractable thematic since last decades whether the induction of autophagy benefits for cell survival or promotes cell death in cerebral ischemia injury. Existing divergent data, like 'muddied the water', bring the complexity and challenge to evaluate the roles of activated autophagy in the pathological process of ischemic stroke. However, like pieces of the puzzle, they also provide clues to objectively understand these opposite outcomes. Growing evidences suggest that the final cell fate partly depends on the game between autophagy demand for substrate removal with cell affordability (autophagy is a adenosine triphosphate (ATP)-dependent process).[39] Thus, it is understandable that many controversial results about the roles of autophagy have been reported in the experiments with different protocols. For better understanding those divergent results, we should consider following factors in data interpretation about the effects played by activated autophagy in ischemic stroke.

\subsection{Potential factors responsible for divergent roles of autophagy}

Table 1 shows controversial results about the effects of autophagy on ischemic brain in literatures with different protocols. Those results appear to be confusing, but opportunity often disguises as challenge. Stark contrast studies described in the Table 1 bring a big challenge to understand the real roles of autophagy in ischemic stroke. On the other hand, they also pave the way for finding the potential factors and 
developing therapeutic strategies for improving outcomes. In the light of this point, herein, we carefully review detail protocols, experimental results and conclusions about the roles of autophagy in those animal models. Following factors should be considered in the experimental designs and results interpretation. 
Table1: Dual roles of autophagy in ischemic brain injury in current literatures

\begin{tabular}{|c|c|c|c|c|c|c|}
\hline Role of autophagy & Model & Treatment & Dose & Timing & Route & Reference \\
\hline Detrimental & $\begin{array}{l}\text { SD rats MCAO } \\
\mathrm{I} 30 \mathrm{~min} / \mathrm{R} 24 \mathrm{~h}\end{array}$ & 3-MA & $600 \mathrm{nmol}$ & at the onset of reperfusion & i.c.v. & {$[40]$} \\
\hline Detrimental & $\begin{array}{l}\mathrm{SD} \text { rats } \mathrm{MCAO} \\
\mathrm{I} 2 \mathrm{~h} / \mathrm{R} 24 \mathrm{~h}\end{array}$ & 3-MA & 400nmol & $\begin{array}{l}\text { 40min before the onset of } \\
\text { reperfusion }\end{array}$ & i.c.v. & [41] \\
\hline Detrimental & $\begin{array}{l}\text { SD rats MCAO } \\
\qquad \mathrm{I} 2 \mathrm{~h} / \mathrm{R} 24 \mathrm{~h}\end{array}$ & 3-MA & $600 \mathrm{nmol}$ & at the onset of reperfusion & i.c.v. & [42] \\
\hline Detrimental & $\begin{array}{l}\text { SD rats MCAO } \\
\qquad \mathrm{I} 2 \mathrm{~h} / \mathrm{R} 72 \mathrm{~h}\end{array}$ & Tat-Beclin-1 & $15 \mathrm{mg}$ & 24 and $48 \mathrm{~h}$ after MCAO & i.p. & [43] \\
\hline Detrimental & SD rats pMCAO & 3-MA & $300 / 600 \mathrm{nmol}$ & 10min after pMCAO & i.c.v. & [44] \\
\hline Detrimental & SD rats pMCAO & $\begin{array}{c}\text { 3-MA } \\
\text { BFA } \\
\text { Z-FA-fmk }\end{array}$ & $\begin{array}{c}\text { 150-600nmoL } \\
4 \mathrm{nmol} \\
13-26 \mathrm{nmol}\end{array}$ & after the onset of pMCAO & i.c.v. & {$[45]$} \\
\hline Detrimental & $\begin{array}{l}\text { C57BL/6 mice } \\
\text { pMCAO }\end{array}$ & $\begin{array}{l}\text { 3-MA } \\
\text { Rapa }\end{array}$ & $\begin{array}{c}60 \mu \mathrm{g} \\
100 \mu \mathrm{g}\end{array}$ & 10min after pMCAO & i.c.v & [46] \\
\hline Detrimental & $\begin{array}{l}\text { C57BL/6 mice } \\
\text { pMCAO }\end{array}$ & 3-MA & $60 \mu \mathrm{g}$ & after the onset of pMCAO & i.c.v. & [47] \\
\hline Detrimental & $\begin{array}{c}\text { SD rats } \\
\text { 20min global } \\
\text { ischemia }\end{array}$ & 3-MA & $600 \mathrm{nmol}$ & $\begin{array}{c}30 \mathrm{~min} / 60 \mathrm{~min} \text { before ischemia } \\
60 \mathrm{~min} \text { after reperfusion }\end{array}$ & i.c.v. & {$[48]$} \\
\hline Detrimental & $\begin{array}{l}\text { SD rats MCAO } \\
\text { I } 2 \mathrm{~h} / \mathrm{R} 24 \mathrm{~h}\end{array}$ & 3-MA & $50 \mathrm{nM}$ & before MCAO & $\begin{array}{l}\text { stereotaxic } \\
\text { injections }\end{array}$ & [49] \\
\hline
\end{tabular}




\begin{tabular}{|c|c|c|c|c|c|c|}
\hline Role of autophagy & Model & Treatment & Dose & Timing & Route & Reference \\
\hline Detrimental & $\begin{array}{r}\text { SD rats MCAO } \\
\mathrm{I} 90 \mathrm{~min} / \mathrm{R} 24 \mathrm{~h}\end{array}$ & $\begin{array}{c}\text { PcDNA plenti6.3-Bec } \\
\text { LY294002 }\end{array}$ & $\stackrel{-}{10 \mathrm{mg} / \mathrm{kg}}$ & 30min before MCAO & $\begin{array}{l}\text { i.c.v. } \\
\text { i.p. }\end{array}$ & {$[18]$} \\
\hline Detrimental & $\begin{array}{c}\text { SD rats MCAO } \\
\mathrm{I} 1 \mathrm{~h} / \mathrm{R}\end{array}$ & 3-MA & 600nmol & 1h before MCAO & i.c.v. & [50] \\
\hline Beneficial & $\begin{array}{l}\text { SD rats MCAO } \\
190 \mathrm{~min} / \mathrm{R} 72 \mathrm{~h}\end{array}$ & LY294002 & $15 \mu \mathrm{M}$ & 30min before MCAO & i.c.v. & [51] \\
\hline Beneficial & $\begin{array}{l}\text { SD rats MCAO } \\
\text { I2h/R22h }\end{array}$ & LY294002 & $15 \mu \mathrm{M}$ & 30min before $\mathrm{MCAO}$ & i.c.v. & [52] \\
\hline Beneficial & $\begin{array}{l}\mathrm{SD} \text { rats MCAO } \\
\mathrm{I} 2 \mathrm{~h} / \mathrm{R} 22 \mathrm{~h}\end{array}$ & $\begin{array}{c}\text { 3-MA } \\
\text { Rapa } \\
\mathrm{Li}_{2} \mathrm{CO}_{3}\end{array}$ & $\begin{array}{c}0.15 \mathrm{mg} / \mathrm{kg} \\
150 \mu \mathrm{g} / \mathrm{kg} \\
20 \mathrm{mg} / \mathrm{kg}\end{array}$ & $0.5 \mathrm{~h}$ before MCAO & i.v. & [53] \\
\hline Beneficial & $\begin{array}{l}\text { SD rats } \\
\text { pMCAO }\end{array}$ & 3-MA & 200nmol & 24h before pMCAO & i.c.v. & {$[54,55]$} \\
\hline Beneficial & $\begin{array}{l}\text { SD rats } \\
\text { pMCAO }\end{array}$ & $\begin{array}{l}\text { 3-MA } \\
\text { BFA } \\
\text { Rapa }\end{array}$ & $\begin{array}{l}100-400 \mathrm{nmol} \\
4 \mathrm{nmol} \\
8.8-35 \mathrm{pmol}\end{array}$ & 24h before pMCAO & i.c.v. & [56] \\
\hline Beneficial & $\begin{array}{c}\text { Mice } \\
\text { pMCAO }\end{array}$ & 3-MA & $60 \mu \mathrm{g}$ & 30min before $\mathrm{MCAO}$ & i.c.v. & [57] \\
\hline Beneficial & $\begin{array}{c}\text { C57BL/6 J mice } \\
\text { MCAL } \\
\text { eMCAO }\end{array}$ & $\begin{array}{c}\text { Rapa } \\
\text { chloroquine }\end{array}$ & $\begin{array}{c}0.625-2.5 \mathrm{mg} / \mathrm{kg} \\
30-90 \mathrm{mg} / \mathrm{kg}\end{array}$ & $\begin{array}{l}\text { after the onset of ischemia; } \\
24 \mathrm{~h} \text { post-stroke }\end{array}$ & i.p. & {$[58]$} \\
\hline
\end{tabular}

(p)MCAO: (permanent) middle cerebral artery occlusion; I: ischemia; R: reperfusion; 3-MA: 3-Methyladenine; i.c.v.: intracerebroventricular; i.p.: intraperitoneal; BFA: bafilomycin A; Rapa: rapamycin; eMCAO: embolic MCAO; MCAL: middle cerebral artery ligation. 


\subsubsection{Therapeutic timing of autophagy regulators}

Autophagy is a dynamic regulatory process and its roles depend on different phases of ischemia. Thus, we screen and analyze relevant studies with similar experimental protocols and pay special attention on the intervening time points of autophagy regulators in both rat models [44, 45, 54-56] and mouse models [46, 47, 57]. It is obvious that administration timing of autophagy regulators could be a critical factor to different outcomes. In other words, therapeutic time points could determine the outcomes of autophagy regulation. Interestingly, the claimed beneficial effects of autophagy on ischemic brain were commonly reported in the literatures with the administration of autophagy inhibitors or activators prior to ischemic insults.[51-57] In contrast, evidences supporting the detrimental roles of autophagy in ischemic brain were often obtained from the literatures using autophagy regulators after the onset of ischemic insults or at the phase of reperfusion.[40-47] Those studies indicate that autophagy activation could be an attempt for survival at early stage of cerebral ischemia, but those self-defensive functions might be pinned down by serious damages attributed by ischemia plus reperfusion. In another word, reperfusion after ischemia might be a turning point transferring autophagic roles from protective to destructive. Importance of intervening timing inspires to select proper therapeutic time point for better outcomes.

\subsubsection{Autophagy inhibitor or activator}

To date, efforts have been made for searching a single silver bullet for autophagy regulation, like finding a fitting shoe for Cinderella.[59] But, no one is perfect yet. For 
autophagy inhibition, it could be executed by pharmacologic inhibitors or knockdown/knockout of specific proteins like Beclin 1, LC3, Atgs of autophagy pathways.[59, 60] It appears that genetic mutation animal models might not be ideal choices for autophagy studies because autophagy is a dynamic process. The mutation for specific genes in autophagy signal pathways would be far beyond the clinical scenario. Actually, PI3K inhibitors, like 3-methyladenine (3-MA), LY294002 and wortmannin could be good tools for autophagy suppression because those agents could inhibit the formation of autophagosomes.[59] Besides, the other common used inhibitors such as bafilomycin A1 [61] and chloroquine [62] were revealed to prevent autophagosome-lysosome fusion to impede autophagy at the later phase. Nevertheless, there is still no an ideal autophagy inhibitor. Let us take 3-MA as an example. As the first generation of autophagy inhibitor, 3-MA has been widely used for autophagy study.[63] However, a recent report claimed that nutrition state had great impacts on the effects of 3-MA. Under nutrient-rich condition, the prolonged treatment of 3-MA (up to $9 \mathrm{~h}$ ) promoted cellular autophagic flux whereas 3-MA reversely inhibited autophagy under nutrient starvation.[64] The dual roles of 3-MA in autophagy might be due to its different temporal effects on class I and class III PI3K. Moreover, 3-MA was also reported to modulate non-autophagic pathway including activation of PKA [65] and inhibition of NF-kB pathway [49]. Therefore, great cautions should be noted in data interpretation when these inhibitors are used for autophagy studies. As for autophagy induction, rapamycin and RAD001 (a rapamycin-derivative), are commonly used to enhance autophagy via inhibiting mTOR.[66] Yet, mTOR 
inhibitors lack specificity which only partially affect mTOR signaling. The mTOR inhibitors could also activate AKT whilst repressing mTOR.[67, 68] Thus, we should be very cautious in data interpretation when those autophagy regulators are used in the experiments.

\subsubsection{Drug dose, therapeutic route and others}

Besides, drug dose and therapeutic route could also lead to controversial results. For example, 3-MA is typically used as an autophagy inhibitor at a working concentration at $5 \mathrm{mM}$. However, a recent study reported that $3-\mathrm{MA}$ at $2.5 \mathrm{mM}$ did not show inhibitory effects on basal level of autophagy, but reversely triggered autophagic flux.[69] Thus, we should pay attention to the concentrations or doses of autophagy regulators used in those studies. With the same drug, the outcomes could be different when different doses are designed for autophagic regulation. It is necessary to further evaluate the roles of autophagy in ischemic stroke experiments with the concentration of 3-MA at 50nM or lower.[49] Besides the drug concentration, administration route might be another factor should be concerned. In most cases, pharmacologic agents were directly injected into cerebral lateral ventricle in order to minimize the side effects and improve the bioavailability. Meanwhile, some cases applied intraperitoneal or intravenous injection for agent administration.[18, 43, 58] Naturally, the effects of drugs on peripheral system must be taken into consideration. Pharmacokinetics and pharmacodynamics of the drugs may also create the potential problems. Thus, it is understandable that regulating autophagy with the same agents could yield different outcomes.[18, 51] Moreover, different cerebral ischemia models with or without 
reperfusion could also induce divergent results.[50, 54, 55] In short, divergent data about the roles of autophagy in ischemic stroke might depend on multiple factors including intervening time point, specificity of autophagy regulators, and administration dose and route, etc.

\subsection{Autophagy and cell death}

It is self-evident that autophagy activation can play different roles in specific ischemia scenarios. Beyond this, knowledge is still limited on how autophagy alters cell fate to "blind alley". Herein, we review the progress of autophagy-induced cell death, providing insights to develop novel therapeutic strategies for ischemic stroke.

Autophagy can induce different forms of cell death under intensive stress condition like cerebral I/R injury. Merely the presence of autophagy in a dying cell does not equate to autophagic cell death.[70] To avoid confusion, the term of 'autophagic cell death' has been redefined by the Nomenclature Committee on Cell Death (NCCD). It was described to be inhibited by genetic mutating at least two molecules of autophagy pathways.[71] Autosis, a novel form of autophagy-dependent and non-apoptotic cell death has been identified, which is characterized by dilated and fragmented ER, ER disappearance, nuclear membrane convolution and focal swelling of the perinuclear space.[72] Furthermore, this type cell death highly depends on $\mathrm{Na}^{+}, \mathrm{K}^{+}$-ATPase.[73] It was revealed that autotic cell death occurred in neurons of hippocampal CA3 area in the absence of apoptosis and necrosis during cerebral hypoxia-ischemia.[73] Presumably, autosis paves the way for us to understand that autophagy has a causative link with cell death instead of only presence in dying cells. 
It appears to be that unfavorable effects of autophagy on ischemic brain are partially attributed by triggering programmed cell death (PCD) to passive cell death.[74] Ischemic stroke is characterized with the interruption of cerebral blood flow to induce brain damage including cell death. Indeed, necrosis and PCD such as apoptosis have been intensively investigated under ischemic insult.[75] Necrosis predominates in the ischemic core of prompt blood flow blockage. It often occurs within several minutes triggered by abrupt ATP depletion. Morphologically, it is distinguished by the features of vacuolation in cytoplasm, plasma membrane breakdown and induction of inflammation.[76] In general, necrosis is considered as an irreversible and uncontrolled form of cell death like "a falling knife". However, the cells in the penumbra of blood flow reduction often initiate PCD in order to minimize cerebral injury.[77] PCD could be interpreted as last attempt for survival in dying cells, which at least provides therapeutic time window to intervene.[78] With the development of new techniques and high-resolution tools, new types of PCD including necroptosis [79], pyroptosis [80] and ferroptosis [81] have been observed and identified. Next, we discuss how autophagy alters cell fate through changing the types of cell death. It is widely accepted that necrosis, apoptosis and activated autophagy often coexist in the penumbral region. Intracellular ATP level plays a determining role in switching cell death phenotypes from apoptosis to necrosis.[82] Of note, autophagy activation aggravates ATP depletion by excessive removal of damaged but still functional mitochondria and its own consumption.[83, 84] Especially, neurons are vulnerable to ATP depletion due to its high energy demand. Thus, autophagy might be a switch in 
the decision between apoptosis and necrosis via ATP consumption in ischemic brain.

\section{Mitophagy in ischemic stroke}

\subsection{Activation of mitophagy-a selective autophagy in ischemic brain}

Mitochondrial autophagy, also termed mitophagy, is identified by autophagosomes around mitochondria, recruiting LC3-II to degrade the depolarized mitochondria.[85] Besides generating more than $60 \%$ of cellular ATP via oxidative phosphorylation, mitochondria are also widely involved in regulating redox signaling and PCD pathways. Therefore, quality control of mitochondria is critical for cellular homeostasis of brain. Under cerebral $\mathrm{I} / \mathrm{R}$ injury, damaged mitochondria can be divided into functionally uneven two parts. Then, the daughter mitochondria with decreased membrane potential are removed by autophagy.[86] As it turns out, mitophagy is of importance to neurons because of their high dependence on mitochondrial function. Multiple signaling pathways and molecules are involved in recognizing damaged mitochondria for selective degradation. Mitophagy could be majorly mediated by PINK1/Parkin pathway in ischemic stroke.[38] In detail, once mitochondrial potential dramatically decreases, PINK1 as a sensor of potential, will translocate from inner mitochondrial membrane to outer mitochondrial membrane (OMM). Subsequently, PINK1 in OMM will further recruit Parkin from cytosol to damaged mitochondria, initiating autophagic machinery. In addition to Parkin-dependent pathway, receptors located in OMM including NIX [87], BNIP3 [88] and FUNDC1 [89], have also been reported to trigger mitochondrial removal by 
autophagosomes via interaction with LIR motif of LC3 under hypoxia condition. Recent studies also indicate that Ambra1, Cardiolipin and SMURF1 could participate in mitophagy through serving as novel receptors to recruit the phagophores expanding to mitochondria.[90-92] Thus, mitophagy is a complex regulatory system to monitor mitochondrial quality for cell survival.

\subsection{Dual roles of mitophagy in cerebral ischemia injury}

Like autophagy, the roles of mitophagy in ischemic brain still remain obscure. Mitophagy activation has been reported in different experimental conditions including middle cerebral artery occlusion (MCAO) animal models and oxygen-glucose deprived (OGD) neuronal and endothelial cell models.[53, 93] It is noteworthy that activated mitophagy is mainly evident in neurons undergoing ischemia [93, 94], although astrocytes are also documented [95]. Mitophagy is essential to brain function via timely eliminating damaged mitochondria, which produce lots of free radicals, release cytochrome $c$ and activate apoptosis pathway.[96, 97] In cerebral ischemia model, rapamycin attenuated cellular dysfunction via activating mitophagy, which was reversed by 3-MA.[98] However, a contrary opinion claimed that mitophagy might exacerbate brain damage under intensive ischemia stress.[38] Like autophagy, activated mitophagy also possesses dual roles in ischemic stroke: physiological level is indispensable for cell survival and excessive level contributes to cell death.[99, 100] Mitophagy must be balanced by mitochondrial biogenesis to meet brain energy demand, or it will be fatal to the brain, at least partly induced by intracellular ATP depletion. To date, the precise roles and regulation of mitophagy in ischemic stroke 
still remain to be further clarified.

There is still no consensus about the roles of mitophagy in cerebral I/R injury. The major limitations of current studies could be attributed to the lack of reliable and specific methods to assess mitophagy and pharmacologic modulators to specifically regulate mitophagy. A recent study revealed that rapamycin treatment before transient MCAO (tMCAO with $2 \mathrm{~h}$ ischemia plus $22 \mathrm{~h}$ reperfusion) attenuated cerebral $\mathrm{I} / \mathrm{R}$ injury via enhancing mitophagy.[98] However, our unpublished data indicated administration of 3-MA and Mdivi-1 (an inhibitor of Drp1) at the reperfusion onset obviously protected brain against ischemia injury in a similar MCAO model through inhibiting mitophagy. These contrary results suggest reperfusion might be a turning point for switching the roles of mitophagy in ischemic brain. More intriguingly, different ischemia periods followed by reperfusion might also induce different mitophagic outcomes. For example, suppression of mitophagy at the onset of reperfusion reinforced neuronal injury in tMCAO model with $1 \mathrm{~h}$ ischemia plus $23 \mathrm{~h}$ reperfusion.[93] Conversely, in another tMCAO model with $6 \mathrm{~h}$ ischemia plus $18 \mathrm{~h}$ reperfusion, treatment of carnosine at the onset of reperfusion attenuated mitophagy, accompanying with the improved brain function.[100] Actually, with the critical importance of mitochondria in brain function, the roles of activated mitophagy in ischemic stroke have gained widespread attention. In the meantime, the big void in precise roles of mitophagy has sparked intensive interests to deeply understand mitophagic process and seek for intervening strategies for therapeutic benefit. Therefore, it is warranted to design series of studies using different cerebral I/R 
protocols and pharmacological/genomic modulation to figure out the turning points and the factors switching beneficial effects to detrimental actions of mitophagy.

\section{Reactive nitrogen species (RNS) and autophagy}

\subsection{Roles of RNS during cerebral I/R injury}

Undoubtedly, modulating autophagy/mitophagy in ischemic stroke will have a potential therapeutic prospect in clinical practice. Reperfusion after ischemia might be a switch to determine different autophagic/mitophagic outcomes. Thus, it is a wise choice to search and regulate key upstream signaling molecules, which majorly form or perform functions during reperfusion to mediate autophagy/mitophagy.

$\mathrm{I} / \mathrm{R}$ insults induce the generation of ROS and RNS, triggering numerous molecular cascades and resulting in brain damage.[25, 101] Thrombolytic therapy is still the most effective therapeutic strategy to rescue the neurons in penumbra.[102] However, reperfusion after ischemia alters microenvironment abruptly, inducing a large number of free radicals and aggravating brain injury.[103] Free radicals are mainly from the dysfunction of mitochondrial oxidative phosphorylation. Damaged but still functional mitochondria generate NADH-supported hydrogen peroxide at a rate up to 10 -fold higher than that of intact mitochondria.[104] It is well known that mitochondria are not only the pools of free radicals, but major targets of oxidative/nitrosative stress. Once oxidative/nitrosative stress overloads cellular antioxidant capacities, it will induce mitochondrial dysfunction.[24] Under physiological level, ROS can serve as important cellular redox signals for preforming various biological functions. However, 
excessive ROS would activate inflammation and trigger over-oxidation to induce brain damage. In autophagy/mitophagy, oxidative stress was thought to decline the mitochondrial membrane potential $(\Delta \Psi \mathrm{m})$ and trigger mitochondrial fission via activating Drp1, playing a critical role in PINK1/Parkin-mediated mitophagy during cerebral I/R injury.[105, 106] Also, ROS were reported to regulate PINK1/Parkin pathway to mediate cadmium-induced mitophagy.[107] The roles of ROS and their underlying regulatory mechanisms during autophagy/mitophagy have been systemically discussed in a recent review article.[108] Thus, in following session, we focus on the roles and regulatory mechanisms of RNS in autophagy/mitophagy during cerebral I/R injury.

As representative $\mathrm{RNS}, \mathrm{NO}$ and $\mathrm{ONOO}^{-}$play critical roles in cell death and $\mathrm{BBB}$ disruption during cerebral I/R injury. In most cases, NO is produced from $L$-arginine and oxygen via enzymatic catalysis by three types of NOS including neuronal NOS (nNOS, type 1), inducible NOS (iNOS, type2), and endothelial NOS (eNOS, type 3). All three NOS subtypes are expressed in the brain. NO at low concentration generated by eNOS and nNOS acts as an indispensable messenger to regulate post- or pre-synaptic activities [109], cerebral blood flow [110] and inflammatory response [111]. Yet, excessive NO derived from iNOS and nNOS could trigger cell death and BBB leakage during I/R injury.[25] Our group has made great efforts to study the roles and mechanisms of NO in neurological deficits, neuronal cell death and BBB disruption in ischemic stroke. In our early study, by using electron paramagnetic resonance spin trapping technology and molecular biology approaches, we found that 
NO generation had two phases in ischemic brain: The first phase of NO was generated from the eNOS activation at ischemia phase but the second phase of NO generation was from the activation and upregulation of iNOS and nNOS at the stage of reperfusion. At reperfusion stage, a large amount of NO was found in both ischemic core and penumbra of the ischemic brain. Besides, non-selective NOS inhibitor $N^{\mathrm{G}}$-nitro-l-arginine methyl ester, nNOS selective inhibitor 7-nitroindazole and iNOS selective inhibitor $1-N_{6}$-(1-iminoethyl)-lysine remarkably reduced infarction volume and brain damage during cerebral I/R injury.[112] Further studies indicate that caveolin-1, a $22 \mathrm{kDa}$ integral membrane protein located at the caveolae, could be critical molecular target of NO.[113] In our recent studies, caveolin-1 was demonstrated to attenuate matrix metalloproteinases (MMPs) activity, prevent tight junction proteins degradation and protect the brain against ischemic injury by inhibiting RNS production.[114-116] Therefore, we propose that RNS/caveolin-1/MMPs signaling cascades could be important molecular mechanisms and therapeutic targets for ischemic stroke.[128-130] Accordingly, selective nNOS inhibitors like tirilazad [117], ARL-17477 [118] and selective iNOS inhibitors such as 1400W [119] and aminoguanidine [120] have the potentials to be drug candidates for ischemic stroke treatment. Nevertheless, it is worthy to mention that NO derived from eNOS contributes to reduce cerebral ischemia injury, at least attributed by maintaining vascular physiological functions.[121, 122] Therefore, the roles of NO in the pathological process of ischemic stroke depend on the level of NO and its different sources. 
Interestingly, $\mathrm{O}_{2}{ }^{--}$and $\mathrm{NO}$ are simultaneously produced during cerebral I/R injury. The reaction of $\mathrm{NO}$ and $\mathrm{O}_{2}^{--}$is extremely fast to generate $\mathrm{ONOO}^{-}$at the diffusion-limited rate $\left(1.6 \times 10^{10}(\mathrm{~mol} / \mathrm{L})^{-1} \mathrm{~s}^{-1}\right) \cdot[23] \mathrm{ONOO}^{-}$, another representative type of RNS, is a crucial cytotoxic mechanism of $\mathrm{O}_{2}{ }^{--}$and $\mathrm{NO}$ in cerebral $\mathrm{I} / \mathrm{R}$ injury. $\mathrm{ONOO}^{-}$can diffuse across biomembranes and even possess higher diffusion capability than $\mathrm{O}_{2}^{-{ }^{-}}$.[123] Akin to $\mathrm{NO}, \mathrm{ONOO}^{-}$at physiological level possesses significant biological functions.[124] But, excessive $\mathrm{ONOO}^{-}$triggers inflammation, lipid membrane peroxidation and induces mitochondrial dysfunction [125], subsequently exacerbating BBB disruption and brain dysfunction [126]. To date, detection of $\mathrm{ONOO}^{-}$is still a challenging task due to the limitation of its short half-life. Thus, 3-nitrotyrosine (3-NT) is often used as the footprint of $\mathrm{ONOO}^{-}$, which is produced from the nitration of free tyrosine or protein tyrosine.[127, 128] Actually, plasma 3-NT level was revealed to have positive correlation with the magnitude of ischemic brain injury in stroke patients.[129] Developing effective tools to monitor the production of $\mathrm{ONOO}^{-}$is critical. Recently, we have successfully developed several novel fluorescent probes for detecting $\mathrm{ONOO}^{-}$with high sensitivity and reliability.[130, 131] With those fluorescent probes, we directly visualized $\mathrm{ONOO}^{-}$ production in the ischemic brain tissues and proved the capacities of several natural compounds to scavenge $\mathrm{ONOO}^{-}$against I/R injury.[132, 133] Targeting $\mathrm{ONOO}^{-}$could be an important and novel strategy for drug development for ischemic stroke. Recent studies by us and others indicated that peroxynitrite decomposition catalysts such as FeTMPyP $[134,135]$ and peroxynitrite scavengers like uric acid [136] could attenuate 
infarct volume, reduce brain edema, prevent BBB leakage and decrease the risk of hemorrhagic transformation in experimental stroke models. Therefore, NO and $\mathrm{ONOO}^{-}$could be critical factors contributing to cerebral I/R injury.

\subsection{Roles of RNS in autophagy/mitophagy}

As mentioned above, reperfusion might be a decision time point to determine different outcomes of autophagy/mitophagy. Coincidentally, a large amount of RNS often trigger series of cascades after reperfusion, contributing to cerebral I/R injury. Further evidences reveal that RNS play a role in regulating autophagy/mitophagy.[137] Thus, we summarize current investigations about the roles of RNS in autophagy/mitophagy.

NO plays its dual roles in autophagy/mitophagy, varying with different cellular context. NO, as an important cellular messenger, serves as regulatory effects via S-nitrosylated proteins (SNO-proteins).[138] Although lots of proteins possess multiple cysteine residues, only specific cysteine residues could be S-nitrosylated. The selectivity of cysteine residues for S-nitrosylation is determined by several molecular factors and mechanisms, like proximal priority.[139] Besides, protein-to-protein transnitrosylation may be another major mechanism to generate SNO-proteins in vivo, during which a NO group is transferred from a donor protein to a selective acceptor protein.[138] Accumulating evidences suggest NO plays a role in modulating autophagy. Nevertheless, there still exists controversy about how NO affects autophagy/mitophagy.[140] One view is that NO activates autophagy/mitophagy, supported by emerging findings. Exposure to 
4-hydroxytamoxifen, NO was proved to be involved in the completion of pro-survival autophagy including autophagic vesicle formation and maturation.[141] Besides, NO participated in physalin A-triggered autophagy in A375-S2 cells via inhibiting mTOR expression.[142] This phenomenon was also observed by another investigation. NO caused nitrosative stress which engaged ATM/LKB1/AMPK/TSC2 signaling cascades to repress mTORC1 and further activate autophagy.[143] In addition, multiple signaling molecules like ERK $[141,144]$ and AMPK $[145,146]$ were demonstrated to involve in NO-induced autophagy. Intriguingly, NO also plays a critical role in mitochondrial fission and removal of damaged mitochondria by autophagy. One study illustrated that the interaction of full-length PINK1 and nNOS triggered Parkin translocation then induced mitophagy. Moreover, it was shown that optimum levels of NO were sufficient for the recruitment of Parkin to the damaged mitochondria, even in PINK1 deficiency.[147]

Of note, there also exist opposite evidences to support that NO suppresses autophagy instead of activating. David C. Rubinsztein's group found NO inhibited autophagy by suppressing the activity of S-nitrosylation substrates, like JNK1 and IKK $\beta$. Overexpression of nNOS, iNOS, or eNOS also blocked autophagosome formation via the JNK1-Bcl-2 pathway.[148] Indeed, growing data support the inhibitory effects of NO in autophagy. NO affects at least two proautophagic pathways: JNK1/Bcl-2/Beclin 1 and IKK $\beta / A M P K / m T O R C 1$ to impair autophagy via SNO-proteins.[140] For examples, NO was revealed to inhibit autophagy through mTOR activation in lipopolysaccharide-stimulated macrophages.[149] Additionally, 
series of studies indicated that NO served as inhibitory effects on autophagy through suppressing JNK1 activity.[150, 151] Nitrosylated Bcl-2 triggered by NO was also reported to inhibit autophagic flux via stabilizing the interaction of $\mathrm{Bcl}-2$ with Beclin-1 in lung epithelial cells.[152] Above data indicate the complex and conflictual effects of NO on autophagy/mitophagy. However, it should be mentioned that a majority of S-nitrosylated target proteins are widely involved in multiple biological activities under NO condition. Moreover, autophagic process often shares molecules with other signaling pathways like apoptosis. Thus, it is not surprising to learn that the effects of NO on autophagy are likely to vary with different cells and models.

As another important component of $\mathrm{RNS}, \mathrm{ONOO}^{-}$has also been reported to regulate autophagy/mitophagy. $\mathrm{ONOO}^{-}$modifies target proteins by the interaction of a nitro group (-NO2) with protein tyrosines, which leads to the change of protein functions.[153] Increased levels of both autophagy and 3-NT were observed in the hepatocytes of old rats following the heat stress, with aggravated mitochondria damage and reduced the expression of heat shock proteins.[154, 155] Alternatively, it was reported that endogenous nitrated nucleotide 8-nitro-cGMP promoted autophagic flux to play a crucial role in cytoprotection against bacteria.[156] Exposure to 3-morpholinosydnonimine (SIN-1), a peroxynitrite donor, increased autophagosome formation and Nrf2 activation contributed to cytoprotection in human umbilical vein endothelial cells.[157]

Overall, above exciting data implicate a causal link between RNS and autophagy/mitophagy regulation. Therefore, it promises a new therapeutic opportunity 
in ischemic stroke via targeting RNS to modulate autophagy/mitophagy.

\subsection{RNS-mediated autophagy/mitophagy under ischemia insult}

Autophagy/mitophagy can be mediated by free radicals via posttranslational modification of biomolecules including proteins, lipids and DNA in ischemic stroke.[158, 159] With growing knowledge about the roles of ROS in initiating autophagy/mitophagy, emphasis has been given to the ROS-induced autophagy/mitophagy in ischemic brain injury.[36, 108] On the contrary, the importance of RNS-mediated autophagy/mitophagy in ischemic brain has yet received enough attention.

At physiological level, NO functions as a ubiquitous second messenger to modulate signal transduction pathways and maintain normal biological activities via protein S-nitrosylation modification in CNS. However, under pathological condition, aberrant SNO-proteins induced by excessive NO often lead to protein misfolding, mitochondrial fragmentation and apoptosis.[139] Current advances suggest that aberrant protein S-nitrosylation is involved in many neurodegenerative diseases.[160] For example, NO induced mitochondrial fission via S-nitrosylation of Drp1, resulting in excessive mitophagy and neuronal cell death.[161-163] In response to nitrosative stress, mitochondrial fission and the autophagosomes engulfing injured mitochondria were observed in young neurons.[163] Alternatively, under prolonged ischemic condition, NO acted as an activator to induce caveolin-1-mediated claudin-5 degradation by triggering autophagy. Moreover, NO scavenger C-PTIO and iNOS inhibitor 1400W obviously suppressed the delivery of claudin-5 for degradation.[164] 
Moreover, iNOS activation also participated in upregulating autophagy of vascular endothelial cells, accompanying with increased apoptosis during I/R injury.[165]

Of note, nitration of tyrosine residues is a chemically distinct redox reaction from S-nitrosylation of cysteine thiol, representing another protein modification via the reaction of tyrosine with $\mathrm{ONOO}^{-}$. Proteomic analysis revealed that nitration-related proteins included metabolism proteins, cytoskeleton proteins, chaperones and carrier proteins in the samples from hypoxia/reoxygenated brain tissues.[166] About 23 proteins tyrosine nitration were identified in $\mathrm{I} / \mathrm{R}$ heart and 10 of them were from mitochondria.[167] Interestingly, mitochondrial proteins appear to be more susceptible to nitration stress.[168] Then, nitrated mitochondrial proteins like voltage-dependent anion channels [169], electron transport chain protein subunit complexes $\mathrm{I}-\mathrm{V}$ and heat shock protein-90 further trigger mitochondrial dysfunction and energy metabolism disorder.[170, 171] Besides, emerging evidence showed that ischemia stress induced tight junction proteins degradation and the underlying mechanism was at least partially contributed by $\mathrm{ONOO}^{-}$-mediated autophagy.[172] Taken together, RNS-mediated autophagy/mitophagy may play an indispensable role in cerebral $\mathrm{I} / \mathrm{R}$ injury. Targeting RNS and its signaling pathways could bring therapeutic benefits for ischemic stroke.

\section{Conclusion}

As elaborated above, growing evidences support that RNS-mediated excessive autophagy/mitophagy contributes to cerebral I/R injury. Indeed, autophagy/mitophagy activation in ischemic stroke has been widely accepted in last decade. Nevertheless, 
there still remains controversy about the beneficial or detrimental roles of activated autophagy/mitophagy in ischemic brain. Accumulating in vivo evidences convey an important message that reperfusion after ischemia may become a critical factor determining autophagy/mitophagy roles from neuroprotection to neurotoxicity. In another word, activated autophagy/mitophagy may aggravate cerebral ischemia injury after reperfusion. In regard to this, modulating autophagy/mitophagy after reperfusion could become a potential therapeutic strategy for ischemic stroke. Strikingly, a large amount of RNS predominate after reperfusion, leading to autophagic/mitophagic flux via S-nitrosylation or nitration modification of macromolecules in ischemic brain. Therefore, RNS would be therapeutic targets to modulate autophagy/mitopahgy in ischemic stroke (Figure1). Without doubt, there is still a long way to go before shedding this new light to clinical practice, yet it shows a potential for future ischemic stroke therapy.

\section{Expert opinion}

Autophagy/mitophagy activation during reperfusion after ischemia contributes to brain damages including neuronal cell death, BBB disruption and neurological deficits. Autophagy/mitophagy activation in ischemic brain has been intensively investigated in numerous studies. Nevertheless, existing contrary results increase the challenge to evaluate real roles of autophagy/mitophagy in ischemic stroke. It is necessary to comprehensively understand autophagy/mitophagy at first. Under strict inclusion criteria, we firstly screen and analyze some reports, in which either pharmacologic or genetic modulation of autophagy/mitophagy has been applied. 
Excitingly, reperfusion after ischemia appears to be a turning point to determine final cell fate. This finding provides a potential to explain these controversial data. In particular, autophagy/mitophagy after reperfusion may shorten the therapeutic time window via accelerating intracellular ATP consumption to induce necrosis. In details, ATP depletion triggers transferring from 'proactive apoptosis' to 'passive necrosis', which exacerbates neuronal injury because of its high dependence on energy supply.[82] Recently, detrimental roles of autophagy in ischemic stroke have got somewhat attention.[21] However, the destructive roles of mitophagy during cerebral I/R injury have not been adequately emphasized. Indeed, damaged mitochondria play a key role in metabolism disorders, energy deficiency and cell death. Correspondingly, mitophagy often occupies a dominant position of autophagy.[97] Thus, it is worthy to pay more attention on the roles of mitophagy in ischemic stroke.

Regard to the importance of autophagic/mitophagic flux in ischemic stroke, it becomes a hot field to seek for critical upstream signal molecules as therapeutic targets. RNS might be critical in cerebral I/R injury through triggering a series of cellular signaling cascades and mediating excessive autophagy/mitophagy. RNS can act as upstream modulators to regulate autophagy/mitophagy via S-nitrosylation or nitration modification of specific proteins.[139, 154] Thus, it is potential to take RNS as novel therapeutic targets for protecting ischemic brain via regulating autophagy/mitophagy. Especially, $\mathrm{ONOO}^{-}$is considered as a critical cytotoxic factor with stronger lipid membrane permeability than its parent free radicals and $\mathrm{ONOO}^{-}$ production could be a critical marker reflecting both oxidative and nitrosative 
stress.[125, 126] Further investigations on the roles of $\mathrm{ONOO}^{-}$and its signaling cascades in autophagy/mitophagy will broaden the understanding of mechanisms about cerebral I/R injury. With our recent breakthrough in developing the advanced and highly sensitive fluorescent probes for $\mathrm{ONOO}^{-}$detection $[130,131]$, we are able to screen active compounds with the properties of scavenging $\mathrm{ONOO}^{-}$and regulating autophagy/mitophagy. Targeting RNS-mediated autophagy/mitophagy and related signaling cascades as therapeutic strategies should be drawn great attention and received high priority in future researches. As analyzed in this review, autophagic intervening time point, specificity of autophagy regulators and so on can affect the results of experimental studies about the effects of autophagy/mitophagy in ischemic stroke. Further investigations with more restrictive protocols are desirable. Importantly, considering the potential inconsistence of human subjects and animal models, great attention should be paid to the clinical investigations on stroke patients. Presumably, screening circulating plasma biomarkers linking RNS-mediated autophagy/mitophagy with the magnitude of ischemic brain injury will bring a new light for clinical practice.

Developing novel therapeutic strategies to reverse the ischemic brain injury should enjoy high priority in future studies. Although many neuroprotective agents have been reported to be effective for ischemic brain in animal experiments, no single drug is approved by FDA with proved clinical efficacy for neuroprotection except t-PA for recanalization. With enormous frustration, attentions have been moved to develop novel therapeutic strategies for brain repair like stem cell therapy. Although emerging 
stem cell-based therapy has been proposed to improve neurological functions in pre-clinical studies [173], yet meta-analysis shows no significant effects in clinical practice [174]. To date, recanalization is still the most effective strategy to rescue neurons and improve clinical outcomes including surgical embolectomy and chemical thrombolysis.[175] Noteworthy, thrombolytic agent t-PA for recanalization has restrict therapeutic time window and the subsequent cerebral I/R injury would worsen brain damage, neurological deficits, and increase hemorrhagic transformation even mortality.[176] Recent studies by us and others provide accumulating evidences to support the roles of RNS in cerebral I/R injury.[126-129, 132, 134-136] RNS-mediated excessive autophagy/mitophagy could be an important mechanism in this pathological process. Thus, targeting RNS to regulate autophagy/mitophagy might be a promising combined therapeutic strategy for thrombolytic treatment to extend the therapeutic time window, improve outcomes and reduce the side effects of t-PA in stroke treatment. Therefore, further investigations on RNS-mediated autophagy/mitophagy would not only bring novel insights into the underlying mechanisms of cerebral I/R injury but also have a bright prospect to develop therapeutic strategies for ischemic stroke.

\section{Declaration of interest}

This work has been supported by grant of the National Natural Science Foundation of China (31570855) and RGC GRF Grant, Hong Kong (No.776512M). The authors have no other relevant affiliations or financial involvement with any organization or 
entity with a financial interest in or financial conflict with the subject matter or materials discussed in the manuscript apart from those disclosed.

\section{Article highlights}

1. Activated autophagy has double-edged roles in ischemic stroke. The beneficial or detrimental effects of activated autophagy on ischemic brain appear to be dependent on the experimental protocols with different intervening time points of autophagy, administration routes and doses of autophagy regulators and so forth.

2. Reperfusion after ischemia appears to act as a critical turning point determining the roles of autophagy from neuroprotection to neurotoxicity via affecting programmed cell death.

3. Mitophagy, as a selective autophagy, predominates in ischemic brain and also plays dual roles in ischemic stroke.

4. RNS-mediated excessive autophagy/mitophagy could be an important cellular event contributing to cerebral $\mathrm{I} / \mathrm{R}$ injury. Targeting $\mathrm{RNS}$ to regulate autophagy/mitophagy might shed new light on the therapies of ischemic stroke. 


\section{References}

1. Boya P, Reggiori F, Codogno P. Emerging regulation and functions of autophagy. Nat Cell Biol 2013 Jul;15(7):713-20.

2. Ohsumi Y. Historical landmarks of autophagy research. Cell Res 2014 Jan;24(1):9-23.

** Panoramic historical review on the autophagy research.

3. Galluzzi L, Pietrocola F, Levine B, et al. Metabolic control of autophagy. Cell 2014 Dec 4;159(6):1263-76.

4. Gallagher LE, Williamson LE, EY. C. Advances in Autophagy Regulatory Mechanisms. Cells 2016;5(2).

5. Nikoletopoulou V, Papandreou ME, Tavernarakis N. Autophagy in the physiology and pathology of the central nervous system. Cell Death Differ 2015 Mar;22(3):398-407.

6. Yamamoto A, Yue Z. Autophagy and its normal and pathogenic states in the brain. Annu Rev Neurosci 2014;37:55-78.

7. Liu H, Qiu H, Xiao Q, et al. Chronic Hypoxia-Induced Autophagy Aggravates the Neuropathology of Alzheimer's Disease through AMPK-mTOR Signaling in the APPSwe/PS1dE9 Mouse Model. J Alzheimers Dis 2015;48(4):1019-32.

8. Pan $\mathrm{T}$, Kondo $\mathrm{S}$, Le $\mathrm{W}$, et al. The role of autophagy-lysosome pathway in neurodegeneration associated with Parkinson's disease. Brain 2008 Aug;131(Pt 8):1969-78.

9. Martin DD, Ladha S, Ehrnhoefer DE, et al. Autophagy in Huntington disease and huntingtin in autophagy. Trends Neurosci 2015 Jan;38(1):26-35.

10. Chen $\mathrm{S}$, Zhang $\mathrm{X}$, Song $\mathrm{L}$, et al. Autophagy dysregulation in amyotrophic lateral sclerosis. Brain Pathol 2012 Jan;22(1):110-6.

11. Menzies FM, Fleming A, Rubinsztein DC. Compromised autophagy and neurodegenerative diseases. Nat Rev Neurosci 2015 Jun;16(6):345-57.

12. Tan CC, Yu JT, Tan MS, et al. Autophagy in aging and neurodegenerative diseases: implications for pathogenesis and therapy. Neurobiol Aging 2014 May;35(5):941-57.

13. Huang Z, Adachi H. Natural Compounds Preventing Neurodegenerative Diseases Through Autophagic Activation. J UOEH 2016 Jun 1;38(2):139-48.

14. Wu H, Niu H, Wu C, et al. The autophagy-lysosomal system in subarachnoid haemorrhage. J Cell Mol Med 2016 Mar 29.

15. Liang K, Zhu L, Tan J, et al. Identification of autophagy signaling network that contributes to stroke in the ischemic rodent brain via gene expression. Neurosci Bull 2015 Aug;31(4):480-90.

16. Mizushima N, Komatsu M. Autophagy: renovation of cells and tissues. Cell 2011 Nov 11;147(4):728-41.

17. Wei K, Wang P, Miao CY. A double-edged sword with therapeutic potential: an updated role of autophagy in ischemic cerebral injury. CNS Neurosci Ther 2012 Nov;18(11):879-86.

* Review of the dual roles of autophagy in ischemic stroke.

18. Zheng $\mathrm{Y}, \mathrm{Hou} \mathrm{J}$, Liu J, et al. Inhibition of autophagy contributes to melatonin-mediated neuroprotection against transient focal cerebral ischemia in rats. J Pharmacol Sci 2014;124(3):354-64.

19. Liu C, Gao Y, Barrett J, et al. Autophagy and protein aggregation after brain ischemia. J Neurochem 2010 Oct;115(1):68-78.

20. Martinet W, Timmermans JP, De Meyer GR. Methods to assess autophagy in situ--transmission electron microscopy versus immunohistochemistry. Methods Enzymol 2014;543:89-114. 
21. Chen W, Sun Y, Liu K, et al. Autophagy: a double-edged sword for neuronal survival after cerebral ischemia. Neural Regen Res 2014 Jun 15;9(12):1210-6.

22. Redmann M, Darley-Usmar V, Zhang J. The Role of Autophagy, Mitophagy and Lysosomal Functions in Modulating Bioenergetics and Survival in the Context of Redox and Proteotoxic Damage: Implications for Neurodegenerative Diseases. Aging Dis 2016 Mar;7(2):150-62.

23. Kim A. A panoramic overview of mitochondria and mitochondrial redox biology. Toxicol Res 2014 Dec;30(4):221-34.

24. Kang J, Pervaiz S. Mitochondria: redox metabolism and dysfunction. Biochem Res Int 2012;2012:896751.

25. Chen XM, Chen HS, Xu MJ, et al. Targeting reactive nitrogen species: a promising therapeutic strategy for cerebral ischemia-reperfusion injury. Acta Pharmacol Sin 2013 Jan;34(1):67-77.

** Extensive review on the roles of reactive nitrogen species in cerebral ischemia-reperfusion injury.

26. Scherz-Shouval R, Elazar Z. Regulation of autophagy by ROS: physiology and pathology. Trends Biochem Sci 2011 Jan;36(1):30-8.

27. Lu Q, Harris VA, Kumar $S$, et al. Autophagy in neonatal hypoxia ischemic brain is associated with oxidative stress. Redox Biol 2015 Dec;6:516-23.

28. Pugsley HR. Quantifying autophagy: Measuring LC3 puncta and autolysosome formation in cells using multispectral imaging flow cytometry. Methods 2016 Jun 1.

29. Katsuragi $Y$, Ichimura $Y$, Komatsu M. p62/SQSTM1 functions as a signaling hub and an autophagy adaptor. FEBS J 2015 Dec;282(24):4672-8.

30. Komatsu M, Ichimura Y. Physiological significance of selective degradation of p62 by autophagy. FEBS Lett 2010 Apr 2;584(7):1374-8.

31. Schlafli AM, Berezowska S, Adams O, et al. Reliable LC3 and p62 autophagy marker detection in formalin fixed paraffin embedded human tissue by immunohistochemistry. Eur J Histochem 2015;59(2):2481.

* LC3 and p62, as autophagy markers, were detected in human tissue by immunohistochemistry.

32. Tian F, Deguchi K, Yamashita T, et al. In vivo imaging of autophagy in a mouse stroke model. Autophagy $2010 \mathrm{Nov} ; 6(8): 1107-14$.

33. Li H, Qiu S, Li X, et al. Autophagy biomarkers in CSF correlates with infarct size, clinical severity and neurological outcome in AIS patients. J Transl Med 2015;13:359.

34. Frugier T, Taylor JM, McLean C, et al. Evidence for the recruitment of autophagic vesicles in human brain after stroke. Neurochem Int 2016 Jun;96:62-8.

* Autophagic vesicles were observed in human post-mortem brain tissue after stroke.

35. Shi R, Weng J, Zhao L, et al. Excessive autophagy contributes to neuron death in cerebral ischemia. CNS Neurosci Ther 2012 Mar;18(3):250-60.

36. Pan R, Timmins GS, Liu W, et al. Autophagy Mediates Astrocyte Death During Zinc-Potentiated Ischemia--Reperfusion Injury. Biol Trace Elem Res 2015 Jul;166(1):89-95.

37. Urbanek T, Kuczmik W, Basta-Kaim A, et al. Rapamycin induces of protective autophagy in vascular endothelial cells exposed to oxygen-glucose deprivation. Brain Res 2014 Mar 17;1553:1-11.

38. Liu K, Sun Y, Gu Z, et al. Mitophagy in ischaemia/reperfusion induced cerebral injury. Neurochem Res 2013 Jul;38(7):1295-300.

39. Kang C, Avery L. To be or not to be, the level of autophagy is the question: dual roles of autophagy in the survival response to starvation. Autophagy 2008 Jan;4(1):82-4. 
40. Gao L, Jiang T, Guo J, et al. Inhibition of autophagy contributes to ischemic postconditioning-induced neuroprotection against focal cerebral ischemia in rats. PLoS One 2012;7(9):e46092.

41. Wang J, Han D, Sun M, et al. A Combination of Remote Ischemic Perconditioning and Cerebral Ischemic Postconditioning Inhibits Autophagy to Attenuate Plasma HMGB1 and Induce Neuroprotection Against Stroke in Rat. J Mol Neurosci 2016 Apr;58(4):424-31.

42. Zhu $Y, B u Q$, Liu $X$, et al. Neuroprotective effect of TAT-14-3-3epsilon fusion protein against cerebral ischemia/reperfusion injury in rats. PLoS One 2014;9(3):e93334.

43. Li L, Tian J, Long MK, et al. Protection against Experimental Stroke by Ganglioside GM1 Is Associated with the Inhibition of Autophagy. PLoS One 2016;11(1):e0144219.

44. Qin AP, Liu CF, Qin YY, et al. Autophagy was activated in injured astrocytes and mildly decreased cell survival following glucose and oxygen deprivation and focal cerebral ischemia. Autophagy 2010 Aug;6(6):738-53.

45. Wen YD, Sheng R, Zhang LS, et al. Neuronal injury in rat model of permanent focal cerebral ischemia is associated with activation of autophagic and lysosomal pathways. Autophagy 2008 Aug;4(6):762-9.

46. Yang Z, Zhong L, Zhong S, et al. Hypoxia induces microglia autophagy and neural inflammation injury in focal cerebral ischemia model. Exp Mol Pathol 2015 Apr;98(2):219-24.

47. Wei N, Yu SP, Gu XH, et al. The involvement of autophagy pathway in exaggerated ischemic brain damage in diabetic mice. CNS Neurosci Ther 2013 Oct;19(10):753-63.

48. Wang JY, Xia Q, Chu KT, et al. Severe global cerebral ischemia-induced programmed necrosis of hippocampal CA1 neurons in rat is prevented by 3-methyladenine: a widely used inhibitor of autophagy. J Neuropathol Exp Neurol 2011 Apr;70(4):314-22.

49. Jiang Y, Zhu J, Wu L, et al. Tetracycline inhibits local inflammation induced by cerebral ischemia via modulating autophagy. PLoS One 2012;7(11):e48672.

50. Xingyong C, Xicui S, Huanxing $S$, et al. Upregulation of myeloid cell leukemia-1 potentially modulates beclin-1-dependent autophagy in ischemic stroke in rats. BMC Neurosci 2013;14:56.

51. Qi Z, Yan F, Shi W, et al. AKT-related autophagy contributes to the neuroprotective efficacy of hydroxysafflor yellow A against ischemic stroke in rats. Transl Stroke Res 2014 Aug;5(4):501-9.

52. Qi Z, Dong W, Shi W, et al. Bcl-2 phosphorylation triggers autophagy switch and reduces mitochondrial damage in limb remote ischemic conditioned rats after ischemic stroke. Transl Stroke Res 2015 Jun;6(3):198-206.

53. Li H, Gao A, Feng D, et al. Evaluation of the protective potential of brain microvascular endothelial cell autophagy on blood-brain barrier integrity during experimental cerebral ischemia-reperfusion injury. Transl Stroke Res 2014 Oct;5(5):618-26.

54. Jiang T, Yu JT, Zhu XC, et al. Acute metformin preconditioning confers neuroprotection against focal cerebral ischaemia by pre-activation of AMPK-dependent autophagy. Br J Pharmacol 2014 Jul;171(13):3146-57.

55. Jiang T, Yu JT, Zhu XC, et al. Ischemic preconditioning provides neuroprotection by induction of AMP-activated protein kinase-dependent autophagy in a rat model of ischemic stroke. Mol Neurobiol 2015 Feb;51(1):220-9.

56. Sheng R, Zhang LS, Han R, et al. Autophagy activation is associated with neuroprotection in a rat model of focal cerebral ischemic preconditioning. Autophagy 2010 May;6(4):482-94. 
57. Wang $\mathrm{P}, \mathrm{Xu}$ TY, Wei $\mathrm{K}$, et al. ARRB1/beta-arrestin-1 mediates neuroprotection through coordination of BECN1-dependent autophagy in cerebral ischemia. Autophagy 2014 Sep;10(9):1535-48.

58. Buckley KM, Hess DL, Sazonova IY, et al. Rapamycin up-regulation of autophagy reduces infarct size and improves outcomes in both permanent MCAL, and embolic MCAO, murine models of stroke. Exp Transl Stroke Med 2014;6:8.

59. Nagelkerke A, Bussink J, Geurts-Moespot A, et al. Therapeutic targeting of autophagy in cancer. Part II: pharmacological modulation of treatment-induced autophagy. Semin Cancer Biol 2015 Apr;31:99-105.

\section{** Recent review about pharmacological modulation of autophagy.}

60. Zhang Z, Guo M, Zhao S, et al. The update on transcriptional regulation of autophagy in normal and pathologic cells: A novel therapeutic target. Biomed Pharmacother 2015 Aug;74:17-29.

61. Mauvezin C, Neufeld TP. Bafilomycin A1 disrupts autophagic flux by inhibiting both V-ATPase-dependent acidification and Ca-P60A/SERCA-dependent autophagosome-lysosome fusion. Autophagy 2015;11(8):1437-8.

62. Zhou J, Hu SE, Tan SH, et al. Andrographolide sensitizes cisplatin-induced apoptosis via suppression of autophagosome-lysosome fusion in human cancer cells. Autophagy 2012 Mar;8(3):338-49.

63. Seglen PO, Gordon PB. 3-Methyladenine: specific inhibitor of autophagic/lysosomal protein degradation in isolated rat hepatocytes. Proc Natl Acad Sci U S A 1982 Mar;79(6):1889-92.

64. Wu YT, Tan HL, Shui G, et al. Dual role of 3-methyladenine in modulation of autophagy via different temporal patterns of inhibition on class I and III phosphoinositide 3-kinase. J Biol Chem 2010 Apr 2;285(14):10850-61.

* Report about dual roles of 3-MA in autophagy.

65. Heckmann $B L$, Yang $X$, Zhang $X$, et al. The autophagic inhibitor 3-methyladenine potently stimulates PKA-dependent lipolysis in adipocytes. Br J Pharmacol 2013 Jan;168(1):163-71.

66. Kim YC, Guan KL. mTOR: a pharmacologic target for autophagy regulation. J Clin Invest 2015 Jan;125(1):25-32.

67. Ji D, Zhang Z, Cheng L, et al. The combination of RAD001 and MK-2206 exerts synergistic cytotoxic effects against PTEN mutant gastric cancer cells: involvement of MAPK-dependent autophagic, but not apoptotic cell death pathway. PLoS One 2014;9(1):e85116.

68. Rubinfeld H, Cohen O, Kammer A, et al. Combination of mTOR Inhibitors Augments Potency while Activating PI3K Signaling in Pituitary Tumors. Neuroendocrinology 2016;103(5):592-604.

69. Zheng $X Y$, Li LJ, Li W, et al. Low concentrations of chloroquine and 3-methyladenine suppress the viability of retinoblastoma cells synergistically with vincristine independent of autophagy inhibition. Graefes Arch Clin Exp Ophthalmol 2015 Dec;253(12):2309-15.

* A good example about different effects of 3-MA on autophagy at different doses.

70. Denton D, Nicolson S, Kumar S. Cell death by autophagy: facts and apparent artefacts. Cell Death Differ 2012 Jan;19(1):87-95.

* Summary about the evidences of autophagic cell death.

71. Galluzzi L, Vitale I, Abrams JM, et al. Molecular definitions of cell death subroutines: recommendations of the Nomenclature Committee on Cell Death 2012. Cell Death Differ 2012 Jan;19(1):107-20. 
72. Liu Y, Levine B. Autosis and autophagic cell death: the dark side of autophagy. Cell Death Differ 2015 Mar;22(3):367-76.

\section{* Lastest review of the autosis.}

73. Liu Y, Shoji-Kawata S, Sumpter RM, Jr., et al. Autosis is a Na+,K+-ATPase-regulated form of cell death triggered by autophagy-inducing peptides, starvation, and hypoxia-ischemia. Proc Natl Acad Sci U S A 2013 Dec 17;110(51):20364-71.

74. Xia M, Meng G, Jiang A, et al. Mitophagy switches cell death from apoptosis to necrosis in NSCLC cells treated with oncolytic measles virus. Oncotarget 2014 Jun 15;5(11):3907-18.

75. Yuan J. Neuroprotective strategies targeting apoptotic and necrotic cell death for stroke. Apoptosis 2009 Apr;14(4):469-77.

76. Shen HM, Codogno P. Autophagy is a survival force via suppression of necrotic cell death. Exp Cell Res 2012 Jul 1;318(11):1304-8.

77. Xu M, Zhang HL. Death and survival of neuronal and astrocytic cells in ischemic brain injury: a role of autophagy. Acta Pharmacol Sin 2011 Sep;32(9):1089-99.

78. Savateev AV, Savateeva-Liubimova TN. [Apoptosis--universal mechanisms of cell death and survival in ischemia and reperfusion: ways to pharmacological control]. Eksp Klin Farmakol 2010 Dec;73(12):44-9.

\section{** Apoptosis provides therapeutic time window to delay cell death.}

79. Fayaz SM, Suvanish Kumar VS, Rajanikant GK. Necroptosis: who knew there were so many interesting ways to die? CNS Neurol Disord Drug Targets 2014 Feb;13(1):42-51.

80. Bergsbaken T, Fink SL, Cookson BT. Pyroptosis: host cell death and inflammation. Nat Rev Microbiol 2009 Feb;7(2):99-109.

81. Speer RE, Karuppagounder SS, Basso M, et al. Hypoxia-inducible factor prolyl hydroxylases as targets for neuroprotection by "antioxidant" metal chelators: From ferroptosis to stroke. Free Radic Biol Med 2013 Sep;62:26-36.

82. Nicotera P, Leist M, Ferrando-May E. Intracellular ATP, a switch in the decision between apoptosis and necrosis. Toxicol Lett 1998 Dec 28;102-103:139-42.

\section{** A critical role of ATP in the change of different cell death types.}

83. Shi RY, Zhu SH, Li V, et al. BNIP3 interacting with LC3 triggers excessive mitophagy in delayed neuronal death in stroke. CNS Neurosci Ther 2014 Dec;20(12):1045-55.

84. Rami A, Kogel D. Apoptosis meets autophagy-like cell death in the ischemic penumbra: Two sides of the same coin? Autophagy 2008 May;4(4):422-6.

85. Dolman NJ, Chambers KM, Mandavilli B, et al. Tools and techniques to measure mitophagy using fluorescence microscopy. Autophagy 2013 Nov 1;9(11):1653-62.

* Introduction about mitophagy detection under fluorescence microscopy.

86. Ikeda Y, Shirakabe A, Brady C, et al. Molecular mechanisms mediating mitochondrial dynamics and mitophagy and their functional roles in the cardiovascular system. J Mol Cell Cardiol 2015 Jan;78:116-22.

87. Ney PA. Mitochondrial autophagy: Origins, significance, and role of BNIP3 and NIX. Biochim Biophys Acta 2015 Oct;1853(10 Pt B):2775-83.

88. Choe SC, Hamacher-Brady A, Brady NR. Autophagy capacity and sub-mitochondrial heterogeneity shape Bnip3-induced mitophagy regulation of apoptosis. Cell Commun Signal 2015;13:37.

89. Liu L, Feng D, Chen G, et al. Mitochondrial outer-membrane protein FUNDC1 mediates hypoxia-induced mitophagy in mammalian cells. Nat Cell Biol 2012 Feb;14(2):177-85. 
90. Yamaguchi O, Murakawa T, Nishida K, et al. Receptor-mediated mitophagy. J Mol Cell Cardiol 2016 Jun;95:50-6.

91. Hamacher-Brady A, Brady NR. Mitophagy programs: mechanisms and physiological implications of mitochondrial targeting by autophagy. Cell Mol Life Sci 2016 Feb;73(4):775-95.

\section{* Recent review of mitophagy.}

92. Saito T, Sadoshima J. Molecular mechanisms of mitochondrial autophagy/mitophagy in the heart. Circ Res 2015 Apr 10;116(8):1477-90.

93. Zhang $X$, Yan $H$, Yuan $Y$, et al. Cerebral ischemia-reperfusion-induced autophagy protects against neuronal injury by mitochondrial clearance. Autophagy 2013 Sep;9(9):1321-33.

94. Yan $\mathrm{H}$, Zhang $\mathrm{X}, \mathrm{Hu} \mathrm{W}$, et al. Histamine $\mathrm{H} 3$ receptors aggravate cerebral ischaemic injury by histamine-independent mechanisms. Nat Commun 2014;5:3334.

95. Motori E, Puyal J, Toni N, et al. Inflammation-induced alteration of astrocyte mitochondrial dynamics requires autophagy for mitochondrial network maintenance. Cell Metab 2013 Dec 3;18(6):844-59.

96. Lin C, Chao H, Li Z, et al. Melatonin attenuates traumatic brain injury-induced inflammation: a possible role for mitophagy. J Pineal Res 2016 Sep;61(2):177-86.

97. Yuan $Y$, Zhang $X$, Zheng $Y$, et al. Regulation of mitophagy in ischemic brain injury. Neurosci Bull 2015 Aug;31(4):395-406.

98. Li Q, Zhang T, Wang J, et al. Rapamycin attenuates mitochondrial dysfunction via activation of mitophagy in experimental ischemic stroke. Biochem Biophys Res Commun 2014 Feb 7;444(2):182-8.

99. Di Y, He YL, Zhao T, et al. Methylene Blue Reduces Acute Cerebral Ischemic Injury via the Induction of Mitophagy. Mol Med 2015;21:420-9.

100. Baek SH, Noh AR, Kim KA, et al. Modulation of mitochondrial function and autophagy mediates carnosine neuroprotection against ischemic brain damage. Stroke 2014 Aug;45(8):2438-43.

\section{** Detrimental roles of excessive mitophagy.}

101. Shirley R, Ord EN, Work LM. Oxidative Stress and the Use of Antioxidants in Stroke. Antioxidants (Basel) 2014;3(3):472-501.

102. Tsivgoulis G, Katsanos AH, Alexandrov AV. Reperfusion therapies of acute ischemic stroke: potentials and failures. Front Neurol 2014;5:215.

103. Gomis M, Davalos A. Recanalization and Reperfusion Therapies of Acute Ischemic Stroke: What have We Learned, What are the Major Research Questions, and Where are We Headed? Front Neurol 2014;5:226.

104. Grivennikova VG, Kareyeva AV, Vinogradov AD. What are the sources of hydrogen peroxide production by heart mitochondria? Biochim Biophys Acta 2010 Jun-Jul;1797(6-7):939-44.

105. Zuo W, Zhang S, Xia CY, et al. Mitochondria autophagy is induced after hypoxic/ischemic stress in a Drp1 dependent manner: the role of inhibition of Drp1 in ischemic brain damage. Neuropharmacology 2014 Nov;86:103-15.

106. Wang Y, Nartiss Y, Steipe B, et al. ROS-induced mitochondrial depolarization initiates PARK2/PARKIN-dependent mitochondrial degradation by autophagy. Autophagy 2012 Oct;8(10):1462-76.

107. Wei X, Qi Y, Zhang $X$, et al. ROS act as an upstream signal to mediate cadmium-induced mitophagy in mouse brain. Neurotoxicology 2015 Jan;46:19-24.

108. Li L, Tan J, Miao Y, et al. ROS and Autophagy: Interactions and Molecular Regulatory Mechanisms. Cell Mol Neurobiol $2015 \mathrm{Jul} ; 35(5): 615-21$. 


\section{* Recent review of the interactions between ROS and autophagy.}

109. Bradley SA, Steinert JR. Nitric Oxide-Mediated Posttranslational Modifications: Impacts at the Synapse. Oxid Med Cell Longev 2016;2016:5681036.

110. Garry PS, Ezra M, Rowland MJ, et al. The role of the nitric oxide pathway in brain injury and its treatment--from bench to bedside. Exp Neurol 2015 Jan;263:235-43.

111. Zanini GM, Cabrales P, Barkho W, et al. Exogenous nitric oxide decreases brain vascular inflammation, leakage and venular resistance during Plasmodium berghei ANKA infection in mice. J Neuroinflammation 2011;8:66.

112. Shen J, Ma S, Chan P, et al. Nitric oxide down-regulates caveolin-1 expression in rat brains during focal cerebral ischemia and reperfusion injury. J Neurochem 2006 Feb;96(4):1078-89.

\section{** Roles of NO in cerebral ischemia-reperfusion injury.}

113. Sato Y, Sagami I, Shimizu T. Identification of caveolin-1-interacting sites in neuronal nitric-oxide synthase. Molecular mechanism for inhibition of NO formation. J Biol Chem 2004 Mar 5;279(10):8827-36.

114. Gu Y, Dee CM, Shen J. Interaction of free radicals, matrix metalloproteinases and caveolin-1 impacts blood-brain barrier permeability. Front Biosci (Schol Ed) 2011;3:1216-31.

115. Gu Y, Zheng G, Xu M, et al. Caveolin-1 regulates nitric oxide-mediated matrix metalloproteinases activity and blood-brain barrier permeability in focal cerebral ischemia and reperfusion injury. J Neurochem 2012 Jan;120(1):147-56.

116. Fu S, Gu Y, Jiang JQ, et al. Calycosin-7-O-beta-D-glucoside regulates nitric oxide /caveolin-1/matrix metalloproteinases pathway and protects blood-brain barrier integrity in experimental cerebral ischemia-reperfusion injury. J Ethnopharmacol 2014 Aug 8;155(1):692-701.

117. Sena E, Wheble P, Sandercock P, et al. Systematic review and meta-analysis of the efficacy of tirilazad in experimental stroke. Stroke $2007 \mathrm{Feb}$;38(2):388-94.

118. O'Neill MJ, Murray TK, McCarty DR, et al. ARL 17477, a selective nitric oxide synthase inhibitor, with neuroprotective effects in animal models of global and focal cerebral ischaemia. Brain Res 2000 Jul 21;871(2):234-44.

119. Perez-Asensio FJ, Hurtado O, Burguete MC, et al. Inhibition of iNOS activity by $1400 \mathrm{~W}$ decreases glutamate release and ameliorates stroke outcome after experimental ischemia. Neurobiol Dis 2005 Mar;18(2):375-84.

120. Sun $M$, Zhao $Y, G u Y$, et al. Neuroprotective actions of aminoguanidine involve reduced the activation of calpain and caspase-3 in a rat model of stroke. Neurochem Int 2010 Mar;56(4):634-41.

121. Li Q, Atochin D, Kashiwagi S, et al. Deficient eNOS phosphorylation is a mechanism for diabetic vascular dysfunction contributing to increased stroke size. Stroke 2013 Nov;44(11):3183-8.

122. Chen J, Cui X, Zacharek A, et al. eNOS mediates T090317 treatment-induced angiogenesis and functional outcome after stroke in mice. Stroke $2009 \mathrm{Jul}$;0(7):2532-8.

123. Ferrer-Sueta G, Radi R. Chemical biology of peroxynitrite: kinetics, diffusion, and radicals. ACS Chem Biol 2009 Mar 20;4(3):161-77.

124. Herold S, Fago A. Reactions of peroxynitrite with globin proteins and their possible physiological role. Comp Biochem Physiol A Mol Integr Physiol 2005 Oct;142(2):124-9.

125. Kuhn DM, Sakowski SA, Sadidi M, et al. Nitrotyrosine as a marker for peroxynitrite-induced neurotoxicity: the beginning or the end of the end of dopamine neurons? J Neurochem 2004 May;89(3):529-36. 
126. Ding R, Chen $Y$, Yang S, et al. Blood-brain barrier disruption induced by hemoglobin in vivo: Involvement of up-regulation of nitric oxide synthase and peroxynitrite formation. Brain Res $2014 \mathrm{Jul}$ 7;1571:25-38.

127. Sun L, Yang L, Fu Y, et al. Capacity of HSYA to inhibit nitrotyrosine formation induced by focal ischemic brain injury. Nitric Oxide 2013 Nov 30;35:144-51.

128. Isobe C, Abe T, Terayama Y. Remarkable increase in 3-nitrotyrosine in the cerebrospinal fluid in patients with lacunar stroke. Brain Res 2009 Dec 11;1305:132-6.

129. Bas DF, Topcuoglu MA, Gursoy-Ozdemir Y, et al. Plasma 3-nitrotyrosine estimates the reperfusion-induced cerebrovascular stress, whereas matrix metalloproteinases mainly reflect plasma activity: a study in patients treated with thrombolysis or endovascular recanalization. J Neurochem 2012 Nov;123 Suppl 2:138-47.

* 3-NT, as a useful tool to monitor oxygen/nitrogen radicals in patients.

130. Yang D, Sun ZN, Peng T, et al. Synthetic fluorescent probes for imaging of peroxynitrite and hypochlorous acid in living cells. Methods Mol Biol 2010;591:93-103.

** Sensitive probes for peroxynitrite detction.

131. Yang D, Wang HL, Sun ZN, et al. A highly selective fluorescent probe for the detection and imaging of peroxynitrite in living cells. J Am Chem Soc 2006 May 10;128(18):6004-5.

132. Gong J, Sun F, Li Y, et al. Momordica charantia polysaccharides could protect against cerebral ischemia/reperfusion injury through inhibiting oxidative stress mediated c-Jun N-terminal kinase 3 signaling pathway. Neuropharmacology 2015 Apr;91:123-34.

133. Gao L, Zhou Y, Zhong W, et al. Caveolin-1 is essential for protecting against binge drinking-induced liver damage through inhibiting reactive nitrogen species. Hepatology 2014 Aug;60(2):687-99.

134. Chen HS, Chen XM, Feng JH, et al. Peroxynitrite Decomposition Catalyst Reduces Delayed Thrombolysis-induced Hemorrhagic Transformation in Ischemia-reperfused Rat Brains. CNS Neurosci Ther 2015 Jul;21(7):585-90.

135. Xu M, Chen X, Gu Y, et al. Baicalin can scavenge peroxynitrite and ameliorate endogenous peroxynitrite-mediated neurotoxicity in cerebral ischemia-reperfusion injury. J Ethnopharmacol 2013 Oct 28;150(1):116-24.

136. Yu ZF, Bruce-Keller AJ, Goodman Y, et al. Uric acid protects neurons against excitotoxic and metabolic insults in cell culture, and against focal ischemic brain injury in vivo. J Neurosci Res 1998 Sep $1 ; 53(5): 613-25$.

137. Montagna C, Rizza S, Maiani E, et al. To eat, or NOt to eat: S-nitrosylation signaling in autophagy. FEBS J 2016 Apr 15.

** Extensive review of S-nitrosylation signaling in autophagy.

138. Nakamura T, Lipton SA. Protein S-Nitrosylation as a Therapeutic Target for Neurodegenerative Diseases. Trends Pharmacol Sci 2016 Jan;37(1):73-84.

139. Nakamura T, Tu S, Akhtar MW, et al. Aberrant protein s-nitrosylation in neurodegenerative diseases. Neuron 2013 May 22;78(4):596-614.

* Roles of aberrant s-nitrosylation signaling in neurodegenerative diseases.

140. Haldar SM, Stamler JS. S-Nitrosylation at the interface of autophagy and disease. Mol Cell 2011 Jul 8;43(1):1-3. 
141. Duan L, Danzer B, Levenson VV, et al. Critical roles for nitric oxide and ERK in the completion of prosurvival autophagy in 4OHTAM-treated estrogen receptor-positive breast cancer cells. Cancer Lett 2014 Oct 28;353(2):290-300.

142. He H, Feng YS, Zang LH, et al. Nitric oxide induces apoptosis and autophagy; autophagy down-regulates NO synthesis in physalin A-treated A375-S2 human melanoma cells. Food Chem Toxicol 2014 Sep;71:128-35.

143. Tripathi DN, Chowdhury R, Trudel LJ, et al. Reactive nitrogen species regulate autophagy through ATM-AMPK-TSC2-mediated suppression of mTORC1. Proc Natl Acad Sci U S A 2013 Aug 6;110(32):E2950-7.

144. Ye YC, Wang HJ, Xu L, et al. Oridonin induces apoptosis and autophagy in murine fibrosarcoma L929 cells partly via NO-ERK-p53 positive-feedback loop signaling pathway. Acta Pharmacol Sin 2012 Aug;33(8):1055-61.

145. Park SY, Park MY, Park HG, et al. Nitric oxide-induced autophagy and the activation of AMPK pathway protect against apoptosis in human dental pulp cells. Int Endod J 2016 Feb 10.

146. Yang JY, Park MY, Park SY, et al. Nitric Oxide-Induced Autophagy in MC3T3-E1 Cells is Associated with Cytoprotection via AMPK Activation. Korean J Physiol Pharmacol 2015 Nov;19(6):507-14.

147. Han JY, Kang MJ, Kim KH, et al. Nitric oxide induction of Parkin translocation in PTEN-induced putative kinase 1 (PINK1) deficiency: functional role of neuronal nitric oxide synthase during mitophagy. J Biol Chem 2015 Apr 17;290(16):10325-35.

148. Sarkar S, Korolchuk VI, Renna M, et al. Complex inhibitory effects of nitric oxide on autophagy. Mol Cell 2011 Jul 8;43(1):19-32.

** First report about the inhibitory effects of nitric oxide on autophagy.

149. Zhang L, Cardinal JS, Bahar R, et al. Interferon regulatory factor-1 regulates the autophagic response in LPS-stimulated macrophages through nitric oxide. Mol Med 2012;18:201-8.

150. Shen C, Yan J, Erkocak OF, et al. Nitric oxide inhibits autophagy via suppression of JNK in meniscal cells. Rheumatology (Oxford) 2014 Jun;53(6):1022-33.

151. Ishima Y, Inoue A, Fang J, et al. Poly-S-nitrosated human albumin enhances the antitumor and antimetastasis effect of bevacizumab, partly by inhibiting autophagy through the generation of nitric oxide. Cancer Sci 2015 Feb;106(2):194-200.

152. Wright C, lyer AK, Kulkarni Y, et al. S-Nitrosylation of Bcl-2 Negatively Affects Autophagy in Lung Epithelial Cells. J Cell Biochem 2016 Feb;117(2):521-32.

153. Radi R. Protein tyrosine nitration: biochemical mechanisms and structural basis of functional effects. Acc Chem Res 2013 Feb 19;46(2):550-9.

154. Oberley TD, Swanlund JM, Zhang HJ, et al. Aging results in increased autophagy of mitochondria and protein nitration in rat hepatocytes following heat stress. J Histochem Cytochem 2008 Jun;56(6):615-27.

155. Swanlund JM, Kregel KC, Oberley TD. Autophagy following heat stress: the role of aging and protein nitration. Autophagy 2008 Oct;4(7):936-9.

156. Ito C, Saito Y, Nozawa T, et al. Endogenous nitrated nucleotide is a key mediator of autophagy and innate defense against bacteria. Mol Cell 2013 Dec 26;52(6):794-804.

157. Mattart L, Calay D, Simon D, et al. The peroxynitrite donor 3-morpholinosydnonimine activates Nrf2 and the UPR leading to a cytoprotective response in endothelial cells. Cell Signal 2012 Jan;24(1):199-213. 
158. Kaminskyy VO, Zhivotovsky B. Free radicals in cross talk between autophagy and apoptosis. Antioxid Redox Signal 2014 Jul 1;21(1):86-102.

159. Lizama-Manibusan B, McLaughlin B. Redox modification of proteins as essential mediators of CNS autophagy and mitophagy. FEBS Lett 2013 Aug 2;587(15):2291-8.

160. Nakamura T, Prikhodko OA, Pirie E, et al. Aberrant protein S-nitrosylation contributes to the pathophysiology of neurodegenerative diseases. Neurobiol Dis 2015 Dec;84:99-108.

161. Cho DH, Nakamura T, Fang J, et al. S-nitrosylation of Drp1 mediates beta-amyloid-related mitochondrial fission and neuronal injury. Science 2009 Apr 3;324(5923):102-5.

162. Nakamura T, Cieplak $P, C$ C DH, et al. S-nitrosylation of Drp1 links excessive mitochondrial fission to neuronal injury in neurodegeneration. Mitochondrion 2010 Aug;10(5):573-8.

163. Barsoum MJ, Yuan H, Gerencser AA, et al. Nitric oxide-induced mitochondrial fission is regulated by dynamin-related GTPases in neurons. EMBO J 2006 Aug 23;25(16):3900-11.

164. Liu J, Weaver J, Jin X, et al. Nitric Oxide Interacts with Caveolin-1 to Facilitate Autophagy-Lysosome-Mediated Claudin-5 Degradation in Oxygen-Glucose Deprivation-Treated Endothelial Cells. Mol Neurobiol 2015 Oct 29.

165. Zhu T, Yao Q, Wang W, et al. iNOS Induces Vascular Endothelial Cell Migration and Apoptosis Via Autophagy in Ischemia/Reperfusion Injury. Cell Physiol Biochem 2016;38(4):1575-88.

166. Peinado MA, Hernandez R, Peragon J, et al. Proteomic characterization of nitrated cell targets after hypobaric hypoxia and reoxygenation in rat brain. J Proteomics 2014 Sep 23;109:309-21.

167. Liu B, Tewari AK, Zhang L, et al. Proteomic analysis of protein tyrosine nitration after ischemia reperfusion injury: mitochondria as the major target. Biochim Biophys Acta 2009 Mar;1794(3):476-85.

* Proteomic report of protein tyrosine nitration after ischemia-reperfusion injury.

168. Vattemi G, Mechref $\mathrm{Y}$, Marini M, et al. Increased protein nitration in mitochondrial diseases: evidence for vessel wall involvement. Mol Cell Proteomics 2011 Apr;10(4):M110 002964.

169. Yang M, Camara AK, Wakim BT, et al. Tyrosine nitration of voltage-dependent anion channels in cardiac ischemia-reperfusion: reduction by peroxynitrite scavenging. Biochim Biophys Acta 2012 Nov;1817(11):2049-59.

170. Teng RJ, Wu TJ, Afolayan AJ, et al. Nitrotyrosine impairs mitochondrial function in fetal lamb pulmonary artery endothelial cells. Am J Physiol Cell Physiol 2016 Jan 1;310(1):C80-8.

171. Elfering SL, Haynes VL, Traaseth NJ, et al. Aspects, mechanism, and biological relevance of mitochondrial protein nitration sustained by mitochondrial nitric oxide synthase. Am J Physiol Heart Circ Physiol 2004 Jan;286(1):H22-9.

172. Han F, Chen YX, Lu YM, et al. Regulation of the ischemia-induced autophagy-lysosome processes by nitrosative stress in endothelial cells. J Pineal Res 2011 Aug;51(1):124-35.

173. Tang YH, Ma YY, Zhang ZJ, et al. Opportunities and challenges: stem cell-based therapy for the treatment of ischemic stroke. CNS Neurosci Ther 2015 Apr;21(4):337-47.

174. Wang Q, Duan F, Wang MX, et al. Effect of stem cell-based therapy for ischemic stroke treatment: A meta-analysis. Clin Neurol Neurosurg 2016 Jul;146:1-11.

* Recent meta-analysis of stem cell-based therapy for ischemic stroke.

175. Ansari S, Rahman M, Waters MF, et al. Recanalization therapy for acute ischemic stroke, part 1 : surgical embolectomy and chemical thrombolysis. Neurosurg Rev 2011 Jan;34(1):1-9.

176. Donnan GA, Davis SM, Parsons MW, et al. How to make better use of thrombolytic therapy in acute ischemic stroke. Nat Rev Neurol 2011 Jul;7(7):400-9. 
Table1: Dual roles of autophagy in ischemic brain injury in current literatures

\begin{tabular}{|c|c|c|c|c|c|c|}
\hline Role of autophagy & Model & Treatment & Dose & Timing & Route & Reference \\
\hline Detrimental & $\begin{array}{l}\text { SD rats } \mathrm{MCAO} \\
\mathrm{I} 30 \mathrm{~min} / \mathrm{R} 24 \mathrm{~h}\end{array}$ & 3-MA & $600 \mathrm{nmol}$ & at the onset of reperfusion & i.c.v. & {$[40]$} \\
\hline Detrimental & $\begin{array}{l}\text { SD rats MCAO } \\
\mathrm{I} 2 \mathrm{~h} / \mathrm{R} 24 \mathrm{~h}\end{array}$ & 3-MA & 400nmol & $\begin{array}{l}\text { 40min before the onset of } \\
\text { reperfusion }\end{array}$ & i.c.v. & {$[41]$} \\
\hline Detrimental & $\begin{array}{l}\text { SD rats MCAO } \\
\mathrm{I} 2 \mathrm{~h} / \mathrm{R} 24 \mathrm{~h}\end{array}$ & 3-MA & $600 \mathrm{nmol}$ & at the onset of reperfusion & i.c.v. & {$[42]$} \\
\hline Detrimental & $\begin{array}{l}\text { SD rats MCAO } \\
\text { I2h/R72h }\end{array}$ & Tat-Beclin-1 & $15 \mathrm{mg}$ & 24 and $48 \mathrm{~h}$ after MCAO & i.p. & {$[43]$} \\
\hline Detrimental & SD rats pMCAO & 3-MA & 300/600nmol & 10min after pMCAO & i.c.v. & [44] \\
\hline Detrimental & SD rats pMCAO & $\begin{array}{c}\text { 3-MA } \\
\text { BFA } \\
\text { Z-FA-fmk }\end{array}$ & $\begin{array}{c}150-600 \mathrm{nmoL} \\
4 \mathrm{nmol} \\
13-26 \mathrm{nmol}\end{array}$ & after the onset of pMCAO & i.c.v. & {$[45]$} \\
\hline Detrimental & $\begin{array}{l}\text { C57BL/6 mice } \\
\text { pMCAO }\end{array}$ & $\begin{array}{l}\text { 3-MA } \\
\text { Rapa }\end{array}$ & $\begin{array}{l}60 \mu \mathrm{g} \\
100 \mu \mathrm{g}\end{array}$ & $10 \mathrm{~min}$ after $\mathrm{pMCAO}$ & i.c.v & {$[46]$} \\
\hline Detrimental & $\begin{array}{l}\text { C57BL/6 mice } \\
\text { pMCAO }\end{array}$ & 3-MA & $60 \mu \mathrm{g}$ & after the onset of pMCAO & i.c.v. & {$[47]$} \\
\hline Detrimental & $\begin{array}{c}\text { SD rats } \\
\text { 20min global } \\
\text { ischemia }\end{array}$ & 3-MA & $600 \mathrm{nmol}$ & $\begin{array}{l}30 \mathrm{~min} / 60 \mathrm{~min} \text { before ischemia } \\
60 \mathrm{~min} \text { after reperfusion }\end{array}$ & i.c.v. & {$[48]$} \\
\hline Detrimental & $\begin{array}{l}\text { SD rats MCAO } \\
\text { I2h/R24h }\end{array}$ & 3-MA & $50 \mathrm{nM}$ & before MCAO & $\begin{array}{l}\text { stereotaxic } \\
\text { injections }\end{array}$ & [49] \\
\hline
\end{tabular}




\begin{tabular}{|c|c|c|c|c|c|c|}
\hline Role of autophagy & Model & Treatment & Dose & Timing & Route & Reference \\
\hline Detrimental & $\begin{array}{l}\text { SD rats MCAO } \\
\mathrm{I} 90 \mathrm{~min} / \mathrm{R} 24 \mathrm{~h}\end{array}$ & $\begin{array}{l}\text { PcDNA plenti6.3-Bec } \\
\text { LY294002 }\end{array}$ & $10 \mathrm{mg} / \mathrm{kg}$ & 30min before $\mathrm{MCAO}$ & $\begin{array}{l}\text { i.c.v. } \\
\text { i.p. }\end{array}$ & {$[18]$} \\
\hline Detrimental & $\begin{array}{l}\text { SD rats MCAO } \\
\text { I1h/R }\end{array}$ & 3-MA & $600 \mathrm{nmol}$ & 1h before MCAO & i.c.v. & {$[50]$} \\
\hline Beneficial & $\begin{array}{l}\text { SD rats MCAO } \\
\text { I90min/R72h }\end{array}$ & LY294002 & $15 \mu \mathrm{M}$ & 30min before $\mathrm{MCAO}$ & i.c.v. & {$[51]$} \\
\hline Beneficial & $\begin{array}{l}\text { SD rats MCAO } \\
\text { I2h/R } 22 \mathrm{~h}\end{array}$ & LY294002 & $15 \mu \mathrm{M}$ & 30min before $\mathrm{MCAO}$ & i.c.v. & {$[52]$} \\
\hline Beneficial & $\begin{array}{l}\text { SD rats MCAO } \\
\qquad \mathrm{I} 2 \mathrm{~h} / \mathrm{R} 22 \mathrm{~h}\end{array}$ & $\begin{array}{c}\text { 3-MA } \\
\text { Rapa } \\
\mathrm{Li}_{2} \mathrm{CO}_{3}\end{array}$ & $\begin{array}{c}0.15 \mathrm{mg} / \mathrm{kg} \\
150 \mu \mathrm{g} / \mathrm{kg} \\
20 \mathrm{mg} / \mathrm{kg}\end{array}$ & $0.5 \mathrm{~h}$ before $\mathrm{MCAO}$ & i.v. & [53] \\
\hline Beneficial & $\begin{array}{l}\text { SD rats } \\
\text { pMCAO }\end{array}$ & 3-MA & 200nmol & 24h before pMCAO & i.c.v. & {$[54,55]$} \\
\hline Beneficial & $\begin{array}{l}\text { SD rats } \\
\text { pMCAO }\end{array}$ & $\begin{array}{l}\text { 3-MA } \\
\text { BFA } \\
\text { Rapa }\end{array}$ & $\begin{array}{c}\text { 100-400nmol } \\
4 \mathrm{nmol} \\
8.8-35 \mathrm{pmol}\end{array}$ & 24h before pMCAO & i.c.v. & {$[56]$} \\
\hline Beneficial & $\begin{array}{c}\text { Mice } \\
\text { pMCAO }\end{array}$ & 3-MA & $60 \mu \mathrm{g}$ & 30min before MCAO & i.c.v. & {$[57]$} \\
\hline Beneficial & $\begin{array}{c}\text { C57BL/6 J mice } \\
\text { MCAL } \\
\text { eMCAO }\end{array}$ & $\begin{array}{c}\text { Rapa } \\
\text { chloroquine }\end{array}$ & $\begin{array}{c}0.625-2.5 \mathrm{mg} / \mathrm{kg} \\
30-90 \mathrm{mg} / \mathrm{kg}\end{array}$ & $\begin{array}{l}\text { after the onset of ischemia; } \\
24 \mathrm{~h} \text { post-stroke }\end{array}$ & i.p. & {$[58]$} \\
\hline
\end{tabular}

(p)MCAO: (permanent) middle cerebral artery occlusion; I: ischemia; R: reperfusion; 3-MA: 3-Methyladenine; i.c.v.: intracerebroventricular; i.p.: intraperitoneal; BFA: bafilomycin A; Rapa: rapamycin; eMCAO: embolic MCAO; MCAL: middle cerebral artery ligation. 


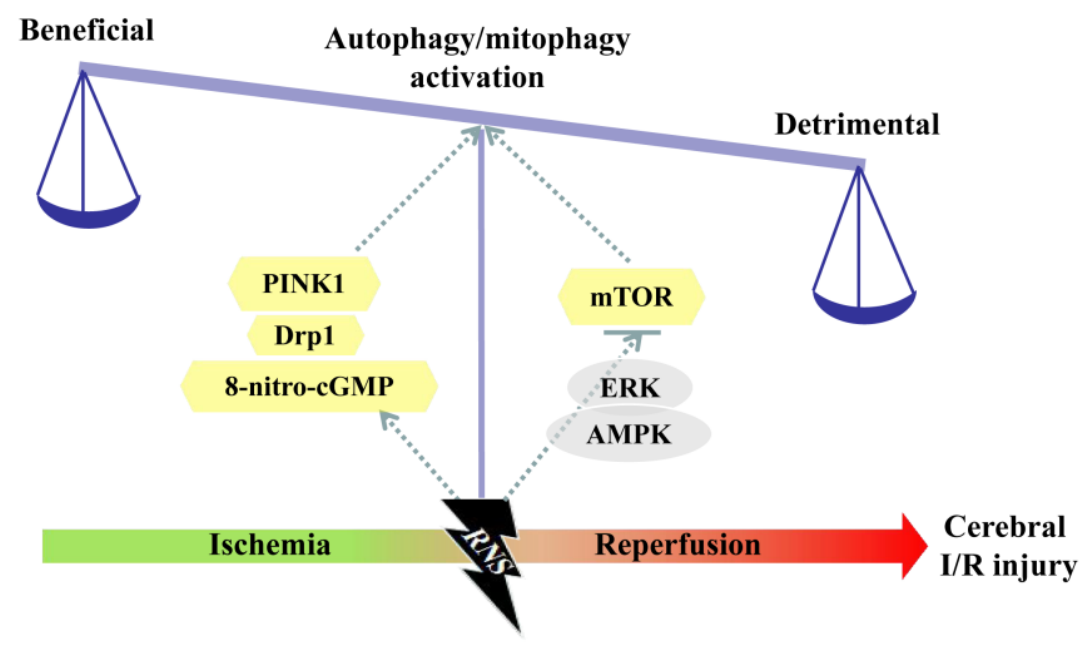

Ischemic stroke 


\section{Legend}

Figure 1. The roles of RNS-mediated autophagy/mitophagy activation in ischemic stroke. A large amount of RNS generate during the reperfusion following cerebral ischemia, triggering numerous molecular cascades and leading to cerebral I/R injury. RNS play critical roles in autophagy/mitophagy activation via protein nitrosylation/nitration modification. In detail, RNS inhibit mTOR expression to activate autophagy through modulating multiple signaling molecules like AMPK and ERK. Moreover, RNS can also trigger autophagy/mitophagy through nitrosylation/nitration modification of related proteins such as Drp1, PINK1 and 8-nitro-cGMP. Activated autophagy/mitophagy has double-edged roles in ischemic stroke. Intriguingly, reperfusion appears to be a decision time point to transfer the roles of activated autophagy/mitophagy from beneficial to detrimental effects. 